\title{
Review \\ Orchidaceae-Derived Anticancer Agents: A Review
}

\author{
Tomasz Śliwiński $^{1}$ (D), Tomasz Kowalczyk ${ }^{2}$ D, Przemysław Sitarek $^{3}$ iD and Marta Kolanowska $^{4,5, *(D)}$
}

Citation: Śliwiński, T.; Kowalczyk, T.; Sitarek, P.; Kolanowska, M Orchidaceae-Derived Anticancer Agents: A Review. Cancers 2022, 14 754. https://doi.org/10.3390/ cancers14030754

Academic Editors: Konstantina Karamanou, Stamatis Rigas and Polydefkis Hatzopoulos

Received: 30 December 2021

Accepted: 28 January 2022

Published: 31 January 2022

Publisher's Note: MDPI stays neutral with regard to jurisdictional claims in published maps and institutional affiliations.

Copyright: (C) 2022 by the authors. Licensee MDPI, Basel, Switzerland. This article is an open access article distributed under the terms and conditions of the Creative Commons Attribution (CC BY) license (https:// creativecommons.org/licenses/by/ $4.0 /)$.
1 Laboratory of Medical Genetics, Faculty of Biology and Environmental Protection, University of Lodz, 90-236 Lodz, Poland; tomasz.sliwinski@biol.uni.lodz.pl

2 Department of Molecular Biotechnology and Genetics, University of Lodz, Banacha 12/16, 90-237 Lodz, Poland; tomasz.kowalczyk@biol.uni.lodz.pl

3 Department of Biology and Pharmaceutical Botany, Medical University of Lodz, Muszynskiego 1, 90-151 Lodz, Poland; przemyslaw.sitarek@umed.lodz.pl

4 Department of Geobotany and Plant Ecology, Faculty of Biology and Environmental Protection, University of Lodz, Banacha 12/16, 90-237 Lodz, Poland

5 Department of Biodiversity Research, Global Change Research Institute AS CR, Bělidla 986/4a, 603 00 Brno, Czech Republic

* Correspondence: marta.kolanowska@biol.uni.lodz.pl

Simple Summary: Orchids are commonly used in folk medicine for the treatment of infections and tumors but little is known about the actual chemical composition of these plants and their anticancer properties. In this paper, the most recent literature on orchid-derived bioactive substances with anticancer properties is reviewed. According to the published data, numerous species of orchids contain potential antitumor chemicals. Still, a relatively insignificant number of species of orchids have been tested for their bioactive properties and most of those studies were on Asian taxa. Broader research, 'including American and African species, as well as the correct identification of samples, is essential for evaluating the usefulness of orchids as a plant family with huge anticancer potential.

Abstract: Species of orchids, which belong to the largest family of flowering plants, are commonly used in folk medicine for the treatment of infections and tumors. However, little is known about the actual chemical composition of these plants and their anticancer properties. In this paper, the most recent literature on orchid-derived bioactive substances with anticancer properties is reviewed. For the assessment, previous papers on the anticancer activity of Orchidaceae published since 2015 were considered. The papers were found by exploring electronic databases. According to the available data, many species of orchids contain potential antitumor chemicals. The bioactive substances in a relatively insignificant number of orchids are identified, and most studies are on Asian taxa. Broader research on American and African species and the correct identification of samples included in the experiments are essential for evaluating the usefulness of orchids as a plant family with vast anticancer potential.

Keywords: orchids; secondary metabolites; anticancer

\section{Introduction}

According to the World Health Organization (WHO) "Guidelines on Safety Monitoring of Herbal Medicines in Pharmacovigilance Systems", up to 80\% of the world's population rely on herbal medicines as a primary source of healthcare. As summarized by Ekor [1], the use of herbal medicines is increasing also in developed countries [2,3]. It is not surprising that the utilization of plants in medicine is as old as mankind itself and even modern pharmacotherapy includes numerous herb-derived drugs [4,5]. Noteworthy, about $10 \%$ of known vascular plants are currently used as therapeutics [6]. In 2019 alone, almost 2000 new species of plants were discovered according to the "State of the World's Plants and Fungi 2020" report, published by the Royal Botanic Gardens Kew. These plants could be potential sources of new phytochemicals that can be used in medicine [7]. 
Cancer ranks as a leading cause of premature death around the globe. In 2020, 19.3 million new cases of cancer cases and almost 10 million cancer deaths were reported worldwide [8]. The most common were female breast tumor (11.7\%), lung cancer $(11.4 \%)$, and colorectal cancer (10.0\%) [8]. Moreover, about a $47 \%$ increase in the yearly diagnosed cancer cases is expected to occur in 2040 [8]. Cancer treatments include various medical procedures, e.g., surgical treatment, radiotherapy, and chemotherapy. However, conventional chemotherapeutic agents can fail as a result of chemoresistance development $[9,10]$ and about $80-90 \%$ of the deaths from cancer are assigned to this resistance [11,12]. Natural products have the potential for overcoming drug resistance [13-16]. Obviously, plants serve as an important source of useful anticancer bioactive compounds and previous records indicated that about $60 \%$ of the currently used antitumor drugs are derived from natural products [17-20].

The aim of the present study is to summarize recent advances in research on orchid metabolites with anticancer properties.

\section{Criteria for Selection of Experimental Papers}

This review includes papers regarding the anticancer activity of orchid compounds published since 2015. The papers were found using electronic databases PubMed/MEDLINE, Scopus, Web of Science, and Google Scholar. The quest terms included Orchidaceae alone and as well as with the following: plant extract, derived compounds, tumor, cancer, lung cancer cells, colon cancer cells, breast cancer cells, prostate cancer cells. Experiments on extracts and secondary metabolites of orchids with in vitro activity against various cancer cell lines were also included in the review. Research published in languages other than English and Spanish or without abstracts in these languages, without full access to the complete text, lacking the identification of the taxon at the species level or without a clear objective and methodology were not analyzed. The removal of duplicates of articles obtained from the electronic databases was followed by the verification of other criteria listed above.

\section{Orchidaceae}

Orchidaceae is one of the largest families of flowering plants with more than 27,000 accepted species [21] and more than 31,000-35,000 species are estimated to exist in total [22,23]. This is a cosmopolitan group growing in almost every habitat except deserts and glaciers. The plants are found above the Arctic Circle, in Patagonia, and even on Macquarie Island $[24,25]$. However, the greatest diversity of orchids is recorded in tropical regions, especially in mountainous areas [26].

Orchids can grow as epiphytes, lithophytes, or terrestrial perennial herbaceous plants that lack any permanent woody structures. Adult plants are mostly able to acquire carbon through photosynthesis, but some taxa are mycoheterotrophic [27]. Orchids are extremely diverse and their growth can be sympodial or monopodial. Many species produce storage organs like bulbs or pseudobulbs. Their flat or pleated leaves may be variously arranged on the stem (alternate, opposite, arranged spirally), or may grow only at the base of the plant. Orchid flowers are extremely diverse, usually zygomorphic, and most often containing both male and female reproductive organs. The outer whorl has three sepals and the inner whorl has three petals; however, one petal (lip) is usually modified and differs in appearance from the other two. A central flower structure called the column comprises both the male (anther) and female (stigma) parts of the flower. The ovary is composed of three carpels.

Orchids are often called "masters of deception" due to the diversity of deceptive mechanisms for attracting pollinators, e.g., generalized food deception, food-deceptive floral mimicry, brood-site imitation, shelter imitation, pseudo antagonism, rendezvous attraction, and sexual deception [28,29]. Many nectar-less orchids mimic other pollinatorrewarding plants [30] or produce various pseudo pollen or pollen-like papillae to lure insects [31]. A large group of species is able even to produce chemicals similar to insect sex 
pheromones [32-35] and this means of pollination, called pseudo copulation, is found only in orchids.

Noteworthy, throughout their life orchids, are associated with mycorrhizal and nonmycorrhizal fungi [36-40]. These endophytes most probably increase or modify the production of plant secondary metabolites [40-44].

Orchids became one of the most popular ornamental plants in the Victorian era and currently, the official global orchid trade is estimated to ca. 72 million specimens per year [45]. These plants are widely used as medicines, food, and as herbs with other cultural values $[46,47]$. Currently vanilla together with salep and chikanda are globally and regionally important food products [45]. Orchids were first used in Chinese traditional medicine [48,49], but they are also popular in Ayurvedic therapies [50] and are commonly used by native tribes in tropical America as well as in Africa [51,52].

Noteworthy, despite a long history of orchid research, scientists are still finding many new species in the tropical areas e.g., [53-55] - only in 2020 more than 100 orchids were described, most of them from South America [56-63] and tropical Asia [64-69]. Unfortunately, the taxonomists did not analyze the chemical properties of their secondary metabolites and their potential medicinal usefulness remains unrecognized.

\subsection{Importance of Symbiosis}

As mentioned before, all orchids are associated with specific mycobiota and different fungi species are found in various plant parts [40,70-73]. Preliminary studies already proved that some of these microorganisms are characterized by antimicrobial activities $[74,75]$ and that interactions of symbiotic fungi with plants contribute to secondary metabolites production .

Unfortunately, the comparative studies on compounds extracted from fungi-infected and in vitro cultivated, fungi-free orchids were not conducted so far. Considering the enormous number of orchid species, their symbionts remain poorly recognized. Noteworthy, most of the experiments on orchid endophytic fungi included only root tissue, $[76,77]$ while in traditional medicine, stems, and leaves are organs usually used for therapies $[43,48,49,78-81]$. The importance of recognition of orchid endophytic fungi for secondary metabolites synthesis and their potential application in medicine were summarized by Sarsaiya et al. [39] and Pant et al. [82].

Interestingly, some of the bioactive compounds were found in an invasive orchid species, Arundina graminifolia, which is an Asian native herb. It would be important to study also the populations of this species which are currently invading Central and South America [83] in the context of the differences in symbiotic mycobiota of non-native plants as well as the similarity of secondary metabolites produced by native and invasive populations. Similarly, the compounds produced by Liparis nervosa which grows in Asia, Africa, and America should be compared with plants collected in various geographical regions.

\subsection{Importance of Taxonomy and Plant Material Preservation}

In this study, as experts in orchid taxonomy [84-87], it is crucial to emphasize the fundamental role of the correct identification of plants for further studies on the usefulness of phytochemicals in cancer therapy [88-90]. The diversity of orchids and superficial similarity of related species often leads to erroneous identification of taxa [91,92]. The detailed studies on various orchids revealed that numerous commonly recognized species are actually species-complexes that include several distinct species [93-95].

Most of the reports reviewed in this paper were on Dendrobium, which is one of the most complicated taxa in terms of species nomenclature and classification [96]. Currently, there are more than 1000 species in this genus, and new species and varieties are described frequently from tropical Asia $[97,98]$. Diagnostic characters which allow to identify particular Dendrobium species are related to flower morphology and therefore plants cannot be correctly classified in the vegetative stage [99]. To further investigate orchids used for treating cancer, it is vitally important that they are correctly identified. Initial identification 
of a plant should not only be confirmed by expert taxonomists but also voucher material further verified and preserved in the form of dried herbarium specimens [100] and preferably complemented with DNA barcodes [101-103]. The molecular identification without properly preserved plant material can be doubtful [104,105]. Unfortunately, the good practices summarized by Bussmann [91] are rarely applied in studies on orchid secondary metabolites, therefore it is not possible to confirm the identification of examined species.

\section{Secondary Metabolites of Orchids}

The basic knowledge on the diversity of orchid secondary metabolites was summarized by Sut et al. [71], Teoh [106], and Pant et al. [82] but the authors of these papers did not present data on the action mechanism of particular secondary metabolites, the importance of symbiotic fungi or other issues related with using orchid-derived biocompounds. Experiments on alkaloids, terpenes, stilbenoids, bibenzyls, phenanthrenes, flavonoids, and polysaccharides isolated from Orchidaceae indicated their potential medical usefulness [106].

Gigantol and batatasin III are the main bibenzyls occurring in orchids with cytotoxic activity $[107,108]$. Phenanthrenes are common metabolites of orchids used in traditional medicine [106]. Many of them are cytotoxic and kill human cancer cell lines and possess antiallergic, antimicrobial, antiinflammatory, antioxidant, antiplatelet, and spasmolytic properties [109]. Antitumour properties are reported for monomeric phenanthrenes, biphenanthrenes, and triphenanthrenes [106]. It is also reported that phenanthroquinone (denbinobin) and dihydroxymethoxy phenanthrene (lusianthridin) are cytotoxic [110-112]. Bibenzyl derivatives of phenanthrenes are effective anti-tumor chemicals [113-115]. Alkaloids are another group commonly isolated from orchidis that are important in a medicinal context [48]. Orchid alkaloids are usually classified either as the pyrrolizidine type or the dendrobine-type [106]. Denbinobin triggers apoptosis of numerous human cancer cell lines [70,109,116,117]. A large number of compounds, estimated to exceed 10000 , are aromatics flavonoids, phenols, and tannins [71]. These chemicals have a broad range of pharmacological activities that involve i.a. antioxidant, antimicrobial, antiinflammatory, antimutagenic, antitumour, and immuno-modulatory activities [106,118-120].

Noteworthy, some of the bioactive compounds can be actually produced by the symbiotic microbes associated with orchids [121].

It should be emphasized that our team is also currently working on the identification of phytochemicals in the orchid species described for the first time and on the determination of their biological properties, including anticancer activity.

\section{Biotechnological Methods for Orchidaceae Family}

As shown, plants of the Orchidaceae family can be a source of many valuable, biologically active compounds that can be widely used as a basis or a supplement to the modern forms of oncological therapy. Plants growing in natural habitats are often the only source of these valuable compounds. Due to the fact that these plants usually do not synthesize large amounts of these compounds, it is very difficult to meet the constantly growing demand for these metabolites. What is more, many species capable of their synthesis are under strict protection. The solution to this problem is the use of biotechnological methods allowing constant access to valuable biomass from in vitro cultivation and, in many cases, increasing the level of their synthesis and accumulation. For this purpose, efficient in vitro propagation protocols have already been developed for many medically valuable orchid species. Such an approach often involves the induction of callus tissue which can then be stimulated to differentiate to give rise to new shoots, or in the case of embryogenic callus, it may be the start of somatic embryos. Pujari et al. described three simple, fast, and economical in vitro tissue culture protocols for Dendrobium ovatum that can be used to develop the right amount of material for biological research in an endangered orchid. Additionally, the authors also demonstrated the enhancement of moscatilin production in the in vitro cultures of this valuable plant [122]. Another type of culture that has found application for the Orchidaceae is the protoplast or thin cell layer (TCL) culture. Vudala 
et al. developed an effective micropropagation protocol for Hadrolaelia grandis with thin cell layer culture systems that can be the starting point for in vitro plant breeding, even on a large scale [123]. Additionally, Brattacharyya et al. developed a protocol for the regeneration of Dendrobium aphyllum, an important therapeutic orchid by the t-TCL method. For this purpose, Murashige and Skooga (MS) medium was supplemented with $15 \mu \mathrm{M}$ meta-topoline along with $10 \mu \mathrm{M}$ TDZ and $10 \mu \mathrm{M} \mathrm{AgNO}$. This combination was found to be the most optimal for shoot proliferation [124]. In addition, an adventitious shoot can also be a valuable strategy, which in a relatively short time, using appropriate growth regulators, allows to multiply valuable plant material. As presented by Mahendran et al. who developed a protocol for induction of direct somatic embryogenesis and subsequent plant regeneration for the medicinally important and endangered plant of Malaxis densiflora. In these in vitro studies, seed-derived protocorm explants were cultured on 1/2 Murashige and Skoog medium with 2,4-D, Picloram, and Dicamba alone or in combination with BAP, $\mathrm{TDZ}$, and $\mathrm{Kn}$. It was shown that the best results were obtained on $1 / 2 \mathrm{MS}$ with $3.39 \mu \mathrm{M}$ of 2,4-D and $6.80 \mu \mathrm{M}$ of TDZ. This protocol is another example of work on the possibility of efficient in vitro culture of human-important members of the Orchidaceae family [125]. Another strategy worth considering, among the sources of extremely valuable compounds, is the cultivation of various tissue and cell cultures in special bioreactors [126,127]. These devices, which allow for the maintenance of plant material in sterile conditions in vitro, often allow the optimization of the entire breeding process, which is extremely important from a technological and economic point of view. Bioreactors ensuring optimal conditions for growth and development by strict control of many key parameters have long been used even on an industrial scale in many other plant families. In addition, the possibility of stimulating production with various physical and chemical factors, combined with genetic modifications in the future, will certainly allow the development of efficient and comprehensive solutions allowing the use of the Orchidaceae family as a kind of mini-factories producing compounds desired in many areas of life.

\section{The Anticancer Activity of Plant Extracts from Orchidaceae}

Extracts of many species of orchids have anticancer properties. Isolates from various plant parts exhibit cytotoxic activity against leukemia and melanoma, as well as against brain, breast, cervical, gastric, liver, and lung cancer cells.

Extracts of several species of Dendrobium (Figure 1) have a cytotoxic effect and inhibit the growth of cervical cancer and glioblastoma brain tumor cells [128-132]. It is hypothesized that polyphenol compounds found in orchid extracts inhibit cancer cells by xenobiotic-metabolizing enzymes altering the metabolic activation of potential carcinogens [133]. On the other hand, flavonoids can modify hormone production and prevent the growth of cancer cells [133]. In contrast, phenolics can interrupt cellular division during the telophase stage of mitosis. These chemicals also affect cell proliferation by reducing the amount of cellular protein, the mitotic index, and colony formation [131]. The ethanolic extract of Dendrobium chrysanthum perturbs cell cycle progression and results in a delay in the growth of cells. It also exerts anticancer activity [129]. A similar situation for extracts of $D$. venustum in which phoyunnanin E triggered apoptosis of lung cancer cells by suppression of survivin [134]. Another Dendrobium species, D. crepidatum, is significantly cytotoxic against both cervical cancer (HeLa) and glioblastoma brain tumor (U251) cell lines [128].

Joshi et al. [130] indicate that Vanda cristata (Figure 1) is both cytotoxic against cervical cancer (HeLa) and glioblastoma brain tumor (U251) cell lines, while Vanda cristata, Pholidota articulate, and Papillionanthe uniflora exhibited significant cytotoxic activity against cervical cancer (HeLa) cells. 


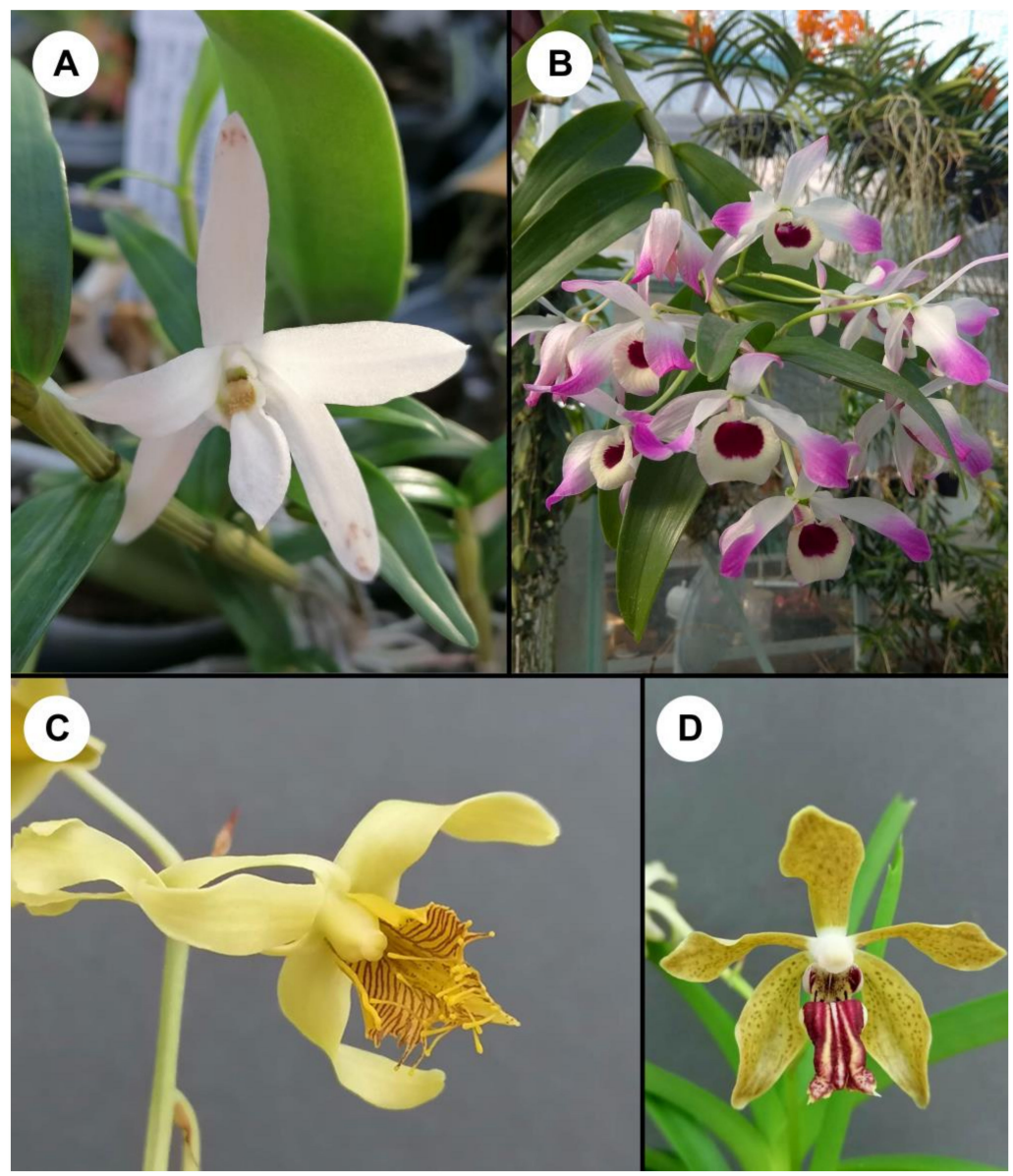

Figure 1. Some orchids with bioactive, antitumor compounds. (A)-Dendrobium moniliforme, (B)_Dendrobium nobile, (C) -Dendrobium venustum, (D) —Vanda cristata

Another promising genus with anticancer properties is Bulbophyllum. B. kwangtungense, and shows antitumour activity against cervical cancer (HeLa) and leukemia (K562) cell lines [130,135]. Bulbophyllum odoratissimum is also cytotoxic against leukemia cell lines (K562, HL-60), hepatoma (BEL-7402), lung adenocarcinoma (A549), and stomach cancer (SGC-7901) cell lines [136]. Extracts of Bulbophyllum sterile bulbs and roots cause apoptosis in human colon cancer (HCT116) cell lines by arresting the G2/M phase of the cell cycle [137].

The volatile oil of Anoectochilus roxburghii induces apoptosis in tumor cells and triggers an enzyme cascade resulting in the apoptosis of lung cancer cells (NCI-H446) [138]. The ethyl acetate extract of Anoectochilus formosanus induces apoptosis in human breast cancer cells (MCF-7) and the aqueous extract effectively inhibits the growth of colon cancer cells in mice [138].

Some studies on Pleione by Liu et al. [139] indicate that an ethyl acetate extract of Pleione bulbocodiodes inhibits the growth of mice cancer cells LA795 (lung adenocarcinoma). Wang et al. [140] also indicate that some components of the extract of Pleione yunnanensis strongly inhibit the growth of lung adenocarcinoma cells. Other compounds obtained from this species are very cytotoxic against colon cancer cells (HepG2), liver cancer cells (BGC-823), and breast cancer cells (MCF-7).

Other orchid extracts that are effective against breast cancer (MCF-7) are those from Eulophia nuda tubers [141], leaves of Aerides odorata [142], and leaves of Vanilla [143]. It is hypothesized that the cytotoxic activity is related to the synergistic action of the phytoconstituents present in these species [141]. Other studies are presented in Table 1. 
Table 1. Cytotoxic effect and potential mechanism of action of Orchid extracts.

\begin{tabular}{|c|c|c|c|c|c|c|}
\hline Name of Species & Part of the Plant & Type of Extract & $\begin{array}{l}\text { Active Compounds/ } \\
\text { Class of Compounds }\end{array}$ & Cancer Cell Lines & $\begin{array}{c}\text { Cytotoxic } \\
\text { Effect/Potential } \\
\text { Mechanism of Action }\end{array}$ & Ref \\
\hline $\begin{array}{c}\text { Acampe praemorsa (Roxb.) } \\
\text { Blatt. \& McCann }\end{array}$ & Leaves & Methanol, ethyl acetate & - & The HeLa and MCF-7 & $\begin{array}{l}\text { Cytotoxic effect (range } \\
49.27-76.94 \mu \mathrm{g} / \mathrm{mL} \text { ) }\end{array}$ & [144] \\
\hline Aeridis odarata Lour. & Leaves & Methanol, ethyl acetate & - & HeLa and MCF-7 & $\begin{array}{c}\text { Cytotoxic effect } \\
\text { (range } 26.21-59.06 \mu \mathrm{g} / \mathrm{mL} \text { ) }\end{array}$ & [144] \\
\hline Eulophia nuda Lindl. & Tubers & Methanol & - & MCF-7 & $\begin{array}{l}\text { Cytotoxic effect } \\
(1000 \mu \mathrm{g} / \mathrm{mL})\end{array}$ & [145] \\
\hline Luisia zeylanica Lindl. & Leaves & $\begin{array}{l}\text { Hexane, chloroform, } \\
\text { ethyl acetate } \\
\text { and methanol }\end{array}$ & $\begin{array}{c}\text { coumarins, flavonoids, glycosides, } \\
\text { phenols, saponins, tannins, } \\
\text { and terpenoids } \\
\text { (2,2-Dimethyl-3-propyloxirane, } \\
\text { Hydroperoxide, 1-ethylbutyl, Ethanone, } \\
\text { 1-cyclohexyl, Cyclopentanol, 1, methyl, } \\
\text { 9,12,15-Octadecatrien-1-ol, } \\
\text { 4-Methyl-1,3-dioxane, } \\
\text { 5-Oxotetrahydrofuran-2- carboxylic } \\
\text { acid, Methyl cis-10-heptadecenoate, } \\
\text { (E)9-Octadecenoic acid ethyl ester, } \\
\text { Triacontane, Methyl } \\
\text { 15-methyl-hexadecanoate€(E)-1-Bis(E)- } \\
\text { but-2-enoxy methoxy but-2-ene, } \\
\text { Kaempferol 3-glucoside, n-Tridecanoic } \\
\text { acid methyl ester, 1, } \\
\text { 4-Dimethyl-1,4,6,7-tetrahydroimidazo 4, } \\
\text { 5-e 1,4 diazepine-5,8-dione, Phthalic } \\
\text { acid, butyl hexyl ester, (Z)-Icos-13-enoic } \\
\text { acid, Octadecanoic acid, ethyl ester }\end{array}$ & MCF-7 and HeLa & $\begin{array}{l}\text { Cytotoxic effect (values } \\
\text { ranging between } \\
18.36 \mu \mathrm{g} / \mathrm{ml} \text { to } \\
67.914 \mu \mathrm{g} / \mathrm{mL} \text { ) }\end{array}$ & [146] \\
\hline $\begin{array}{c}\text { Vanda Tessellata Hook. } \\
\text { Ex G.Don }\end{array}$ & Roots & $\begin{array}{l}\text { Methanol n-hexane and } \\
\text { ethyl acetate }\end{array}$ & $\begin{array}{l}\text { flavonoids, tannins, } \\
\text { saponins, terpenoids, steroids } \\
\text { and alkaloids }\end{array}$ & Hep-G2 and B16-F10 & $\begin{array}{l}\text { Cytotoxic effect (range } \\
\text { 95.3-145.9 } \mu \mathrm{g} / \mathrm{mL} \text { ) }\end{array}$ & [147] \\
\hline Acampe praemorsa (Roxb.) & Leaves & Ethanol & - & A549 & - & [148] \\
\hline
\end{tabular}


Table 1. Cont.

\begin{tabular}{|c|c|c|c|c|c|c|}
\hline Name of Species & Part of the Plant & Type of Extract & $\begin{array}{l}\text { Active Compounds/ } \\
\text { Class of Compounds }\end{array}$ & Cancer Cell Lines & $\begin{array}{c}\text { Cytotoxic } \\
\text { Effect/Potential } \\
\text { Mechanism of Action }\end{array}$ & Ref \\
\hline $\begin{array}{l}\text { Dendrobium officinale } \\
\text { Kimura et. Migo }\end{array}$ & Whole plant & Ethanol & polysaccharide & MCF-7 & $\begin{array}{l}\text { (the possible mechanism } \\
\text { may be that, extract } \\
\text { up-regulates the } \\
\text { LC3-II expression, } \\
\text { down-regulates the } \\
\text { LC3-I expression and } \\
\text { p62 expression. In } \\
\text { addition, extract } \\
\text { inhibits the expression of } \\
\text { PI3K and Akt and their } \\
\text { phosphorylation, and } \\
\text { promotes the expression } \\
\text { of PTEN) }\end{array}$ & [149] \\
\hline Eulophia nuda Lindl. & Tubers & $\begin{array}{l}\text { Alcohol, hydro alcoholic } \\
\text { and aqueous }\end{array}$ & - & MCF-7 & $\begin{array}{l}\text { Cytotoxic effect } \\
(285.1 \mu \mathrm{g} / \mathrm{mL})\end{array}$ & [150] \\
\hline $\begin{array}{l}\text { Arachnis flos-aeris (L.) } \\
\text { Rchb. f. }\end{array}$ & $\begin{array}{l}\text { Leaves, stem } \\
\text { and roots }\end{array}$ & $\begin{array}{l}\text { Chloroform, methanol, } \\
\text { and aqueous }\end{array}$ & terpenoid and phenolic & T47D & $\begin{array}{c}\text { Cytotoxic effect } \\
(139-1436 \mu \mathrm{g} / \mathrm{mL})\end{array}$ & [151] \\
\hline Phaius mishmensis Rchb. & Whole plants & $\begin{array}{l}\text { n-hexane, chloroform, } \\
\text { and ethyl acetate }\end{array}$ & - & $\begin{array}{l}\text { MCF-7, NCI-H460, } \\
\text { and SF-268 }\end{array}$ & $\begin{array}{l}\text { Cytotoxic effect } \\
(1-134 \mu \mathrm{g} / \mathrm{mL})\end{array}$ & [152] \\
\hline $\begin{array}{l}\text { Dendrobium candidum } \\
\text { Wall. ex Lindl. }\end{array}$ & Whole plant & Methanol & - & $\begin{array}{l}\text { SMMC-7721 } \\
\text { and BEL-7404 }\end{array}$ & $\begin{array}{l}\text { Cytotoxic effect (about } \\
1 \mathrm{mg} / \mathrm{mL} \text { ) } \\
\text { It is likely that this } \\
\text { extract suppressed cell } \\
\text { growth by activating } \\
\text { mitochondria apoptosis } \\
\text { pathway and inducing } \\
\text { inhibition of } \\
\text { Wnt/ } \beta \text {-catenin pathway }\end{array}$ & [153] \\
\hline
\end{tabular}


Table 1. Cont.

\begin{tabular}{|c|c|c|c|c|c|c|}
\hline Name of Species & Part of the Plant & Type of Extract & $\begin{array}{l}\text { Active Compounds/ } \\
\text { Class of Compounds }\end{array}$ & Cancer Cell Lines & $\begin{array}{c}\text { Cytotoxic } \\
\text { Effect/Potential } \\
\text { Mechanism of Action }\end{array}$ & Ref \\
\hline $\begin{array}{l}\text { Dendrobium candidum } \\
\text { Wall. ex Lindl. }\end{array}$ & Whole plant & - & - & MCF-7 & $\begin{array}{l}\text { Cytotoxic effect } \\
\quad(2 \mathrm{mg} / \mathrm{mL}) \\
\text { Extract decreased the cell } \\
\text { viability of MCF-7 cells } \\
\text { by inducing cell cycle } \\
\text { arrest at the G2/M phase } \\
\text { and regulating the } \\
\text { key biomarkers }\end{array}$ & [154] \\
\hline $\begin{array}{l}\text { Dendrobium crepidatum } \\
\text { Lindl. \& Paxton and } \\
\text { Dendrobium chrysanthum } \\
\text { Wallich ex Lindley }\end{array}$ & Leaves & Ethanol & - & $\begin{array}{l}\text { Dalton's lymphoma } \\
\text { (DL), a murine } \\
\text { transplantable } \\
\text { T-cell lymphoma }\end{array}$ & $\begin{array}{l}\text { Cytotoxic effect of } \\
\text { extract of } D \text {. crepidatum } \\
\text { at } 325 \mu \mathrm{g} / \mathrm{mL} \text {, while that } \\
\text { for the extract of } D \text {. } \\
\text { chrysanthum was } \\
400 \mu \mathrm{g} / \mathrm{mL} \text {. }\end{array}$ & [155] \\
\hline $\begin{array}{c}\text { Anoectochilus } \\
\text { formosanus Hayata }\end{array}$ & Whole plants & Methanol & - & SCC-25 & $\begin{array}{l}\text { Cell viability assay } \\
\text { indicates that } 1 \mathrm{mg} / \mathrm{mL} \\
\text { extract inhibited oral } \\
\text { cancer SCC- } 25 \text { cell } \\
\text { proliferation by more } \\
\text { than } 82 \%\end{array}$ & [156] \\
\hline Malaxis rheedii Sw. & Whole plants & Methanol & - & MCF-7 and HeLa & $\begin{array}{l}\text { Cytotoxic effect (value of } \\
\text { M. rheedii on MCF-7 cells } \\
\text { was } 167.76 \mu \mathrm{g} / \mathrm{mL} \text { ) }\end{array}$ & [157] \\
\hline
\end{tabular}




\section{The Anticancer Effect-Potential Mechanism of Action and Activation of Signalling Pathways of Pure Compounds from Orchids}

Several classes of phytoconstituents of great chemical diversity have been isolated from therapeutically-used orchids [71].

Various stilbene-based derivatives from orchids, e.g., pholidonone [158], bletilols [159] are cytotoxic against cancer cell lines. The former compound triggers apoptotic cell death in human gastric cancer cells, by inducing ER stress, probably via PERK and IRE1 $\alpha$ signalling pathways [158].

Another group of orchid metabolites that have antitumor activities are phenanthropyrans and phenanthrenes [160-166]. Nudol isolated from Dendrobium nobile arrests the cell cycle of osteosarcoma (U2OS) cells, induces cell apoptosis via the caspase-dependent pathway and suppresses the migration of these cells [161]. Cypripedin isolated from Dendrobium densiflorum is effective against lung cancer by activating caspase- 3 and downregulating the antiapoptotic proteins Bcl-2 and Bcl-xL in cells [167]. Denbinobin also isolated from Dendrobium and Ephemerantha also promotes caspase-3 activity in lung adenocarcinoma cells [168,169] and a polysaccharide extracted from Anoectochilus roxburghii inhibits in this way the growth and proliferation of human prostate cancer (PC-3) cells [138].

Spiranthesphenanthrene isolated from Spiranthes sinensis is cytotoxic against gastric cancer (SGC-7901), hepatocellular carcinoma (HepG2), and melanoma tumor (B16-F10) cell lines [170]. Moreover, this compound significantly inhibits the migration of melanoma tumor (B16-F10) cancer cells [170].

Bulbocodioidins extracted from Pleione bulbocodiodes, which are phenanthrene and phenanthrene/bibenzyl atropisomers, and according to Wang et al. [171], are cytotoxic activity against colon cancer (HCT-116), liver cancer (HepG2), and breast cancer (MCF-7) cell lines. Previously the bibenzyls isolated from this plant were shown to significantly inhibit the growth of leukemia cells (K562, HL-60), liver cancer cells (BEL-7402), gastric cancer cells (SGC-7901), lung cancer cells (A569, H460), and melanoma cells (M14).

Isoviolanthin isolated from Dendrobium officinale reverses TGF- $\beta 1$-mediated epithelialmesenchymal transition in hepatocellular carcinoma (HCC) cells by deactivating the TGF$\beta /$ Smad and PI3K/Akt/mTOR signalling pathways [172].

Phenanthrene and bibenzyl derivatives isolated from Cremastra appendiculata are cytotoxic against colon cancer (HCT-116), liver cancer (HepG2), stomach cancer (BGC-823), lung cancer (A549), and glioma cancer (U251) cell lines [163,173].

One of the most extensively studied orchid compounds is dendrobine and its derivatives $[174,175]$. This chemical induces apoptotic cell death via a mitochondrial-mediated pathway in lung cancer cells (A549). The combination of dendrobine with cisplatin enhances their cytotoxicity by stimulating JNK/p38 stress signalling pathways and, consequently, inducing apoptosis involving the pro-apoptotic proteins Bax and Bim [176].

ViceninII, which is flavonoid glycoside extracted from Dendrobium officinale, inhibits transforming growth factor- $\beta 1$ (TGF- $\beta 1$ )-induced epithelial-mesenchymal transition (EMT) by deactivating TGF- $\beta$ /Smad and PI3K/Akt/mTOR signalling pathways in lung adenocarcinoma A549 and H1299 cells [177].

Recent experiments indicate that erianin isolated from Dendrobium induces ferroptotic cell death in lung cancer cells (H460 and H1299). This action is accompanied by ROS accumulation, lipid peroxidation, and GSH depletion [178,179]. Other research examples are presented in Table 2. 
Table 2. Cytotoxic effect and potential mechanism of action of pure compounds or fractions from Orchids.

\begin{tabular}{|c|c|c|c|c|c|}
\hline Name of Species & Part of the Plant & Active Compounds/Isolated & Cancer Cell Line & $\begin{array}{c}\text { Cytotoxic Effect/ } \\
\text { Mechanism of Action }\end{array}$ & Ref \\
\hline $\begin{array}{l}\text { Dendrobium signatum } \\
\text { Rchb. f. }\end{array}$ & Whole plant & $\begin{array}{l}\text { 3,4-dihydroxy-3,4-dimethoxybibenzyl, } \\
\text { dendrocandin B, dendrocandin I } \\
\text { and dendrofalconerol }\end{array}$ & MDA-231, HepG2 and HT-29 & Cytotoxic effect $(25.2-137.8 \mu \mathrm{M})$ & [180] \\
\hline $\begin{array}{c}\text { Cymbidium } \\
\text { finlaysonianum Lindl }\end{array}$ & $\begin{array}{l}\text { Whole plants } \\
\text { (without flowers) }\end{array}$ & $\begin{array}{c}\text { 1-(4-Hydroxybenzyl)-4,6-dimethoxy- } \\
\text { 9,10-dihydrophenanthrene-2,7-Diol, } \\
\text { ephemeranthoquinone } \\
\text { B, flavanthridin, } \\
\text { 2,4-dimethoxyphenanthrene- } \\
\text { 3,7-diol, 3,4,6- } \\
\text { trimethoxyphenanthrene-2,7-diol, } \\
\text { coelonin, lusianthridin, cymbinodin-A }\end{array}$ & NCI-H187 & Cytotoxic effect $(3.73 \mu \mathrm{M})$ & [181] \\
\hline Dendrobium nobile Lindl. & Stems & $\begin{array}{c}\text { Dendroside, isorhamentin-3-O- } \beta \text {-d- } \\
\text { rutinoside, adenosine, } \\
\text { 4-methoxy-2,5,9R-trihydroxy-9,10- } \\
\text { dihydrophenanthrene } \\
\text { 2-O- } \beta \text {-d-glucopyranoside, }(7 \mathrm{~S}, 8 \mathrm{R}) \\
\text { dehydrodiconiferyl alcohol } \\
9^{\prime}-\beta \text {-glucopyranoside, } \\
\text { koaburaside, uniperoside, } \\
\text { dehydrodiconiferylalcohol-4- } \beta \text { - } \\
\text { d-glucoside }\end{array}$ & HeLa, MCF-7 and A549 & $\begin{array}{c}\text { Cytotoxic effect } \\
\text { (from } 16.8 \text { to }>100 \mu \mathrm{M} \text { ) }\end{array}$ & [182] \\
\hline $\begin{array}{l}\text { Dendrobium williamsonii J. Day } \\
\text { \& Rchb. f. }\end{array}$ & Whole plants & $\begin{array}{c}\text { Aloifol I, moscatilin, } \\
\text { moniliformine, balanophonin, }\end{array}$ & HL-60 & $\begin{array}{l}\text { Cytotoxic effect } \\
(4.48 \text { to } 11.04 \mu \mathrm{M})\end{array}$ & [183] \\
\hline Liparis nervosa (Thunb.) Lindl. & Whole plant & Nervosine VII (alkaloid) & HCT116 & $\begin{array}{l}\text { Nervosine VII simultaneously } \\
\text { induced autophagy and } \\
\text { apoptosis by activating MAPKs } \\
\text { signalling pathway including } \\
\text { JNK, ERK1/2 and p38, } \\
\text { suppressing the p53 } \\
\text { signalling pathway }\end{array}$ & [184] \\
\hline
\end{tabular}


Table 2. Cont.

\begin{tabular}{|c|c|c|c|c|c|}
\hline Name of Species & Part of the Plant & Active Compounds/Isolated & Cancer Cell Line & $\begin{array}{c}\text { Cytotoxic Effect/ } \\
\text { Mechanism of Action }\end{array}$ & Ref \\
\hline $\begin{array}{c}\text { Dendrobium officinale Kimura } \\
\text { et. Migo }\end{array}$ & Leaves & $\begin{array}{c}\text { Denofficin, dendrocandin B, } \\
\text { dendrocandin U, 3,4-dihydroxy- } \\
\text { 5,4'-dimethoxy bibenzyl, moscatilin, } \\
\text { 4,4'-dihydroxy-3,5-dimethoxy } \\
\text { Bibenzyl, gigantol }\end{array}$ & HeLa & Cytotoxic effect (8.0 to $92.4 \mu \mathrm{M})$ & [185] \\
\hline Liparis nervosa (Thunb.) Lindl. & $\begin{array}{c}\text { The whole plants } \\
\text { with roots }\end{array}$ & $\begin{array}{c}\text { Liparisphenanthrenes A, } \\
2,7,2^{\prime} \text {-trihydroxy- } \\
4,4^{\prime}, 7^{\prime} \text {-trimethoxy- } 1,1^{\prime} \text { - } \\
\text { biphenanthrene, } 2,2^{\prime} \text {-dihydroxy- } \\
4,4^{\prime}, 7,7^{\prime} \text {-tetramethoxy- } \\
1,1^{\prime} \text {-biphenanthrene }\end{array}$ & HGC-27 and HT-29 & $\begin{array}{c}\text { Cytotoxic effect } \\
(8.21-9.95 \mu \mathrm{mol} / \mathrm{L}) \\
(8.53-9.27 \mu \mathrm{mol} / \mathrm{L})\end{array}$ & [186] \\
\hline $\begin{array}{l}\text { Paphiopedilum callosum } \\
\text { (Rchb.f.) }\end{array}$ & Roots & $\begin{array}{c}\text { 3'-hydroxy-2,6,5'-trimethoxystilbene, } \\
3^{\prime} \text { - hydroxy-2,5'- } \\
\text { dimethoxystilbene, galangin, } \\
\text { 2,3'-dihydroxy-5'-methoxystilbene }\end{array}$ & MCF-7 and NCI-H187 & $\begin{array}{l}\text { Cytotoxic effect } \\
(62.82-182.48 \mu \mathrm{M})\end{array}$ & [187] \\
\hline $\begin{array}{c}\text { Dendrobium thyrsiflorum } \\
\text { Rchb.f. }\end{array}$ & Whole plants & $\begin{array}{c}\text { 2,7-Dihydroxy-4- } \\
\text { methoxyphenanthrene, } \\
\text { 2,7-Dihydroxy-4-methoxy-9- } \\
\text { fluorenone, } \\
\text { 2,3,5-Trihydroxy-4- } \\
\text { methoxyphenanthrene, } \\
\text { 3,7-Dihydroxy-2,4- } \\
\text { dimethoxyphenanthrene, } \\
\text { 2,7-Dihydroxy-1,5,6- } \\
\text { trimethoxyphenanthrene, } \\
\text { 2,5-Dihydroxy-3,4,9- } \\
\text { trimethoxyphenanthrene, } \\
\text { 2,3,5-Trihydroxy-4-methoxy-9,10- } \\
\text { dihydrophenanthrene, Dengibsin, } \\
\text { Denchrysan B, 2,5-Dihydroxy-4- } \\
\text { methoxy-9,10-dihydrophenanthrene }\end{array}$ & HL-60 and BEL-7402 & $\begin{array}{l}\text { Cytotoxic effect } \\
(1.61 \text { to } 17.25 \mu \mathrm{M})\end{array}$ & [188] \\
\hline $\begin{array}{l}\text { Dendrobium brymerianum } \\
\text { Rchb.f. }\end{array}$ & Whole plant & $\begin{array}{l}\text { moscatilin, gigantol, lusianthridin, } \\
\text { and dendroflorin }\end{array}$ & $\mathrm{H} 460$ & $\begin{array}{c}\text { Cytotoxic effect }(196.7,23.4,65.0 \\
\text { and } 125.8 \mu \mathrm{g} / \mathrm{mL})\end{array}$ & [189] \\
\hline
\end{tabular}


Table 2. Cont.

\begin{tabular}{|c|c|c|c|c|c|}
\hline Name of Species & Part of the Plant & Active Compounds/Isolated & Cancer Cell Line & $\begin{array}{c}\text { Cytotoxic Effect/ } \\
\text { Mechanism of Action }\end{array}$ & Ref \\
\hline $\begin{array}{l}\text { Paphiopedilum godefroyae } \\
\text { (God.-Leb.) Stein }\end{array}$ & Roots & $\begin{array}{c}\text { 2-(3',5'-dimethoxyphenyl)- } \\
\text { 6-hydroxy-5-methoxybenzofuran, } \\
\text { 3-hydroxy-2,5'-dimethoxystilbene, } \\
\text { 2-(E)-2-(3,5-dimethoxyphenyl)- } \\
\text { vinyl-phenol, } \\
\text { 5,6-dimethoxy-2-(3-hydroxy-5- } \\
\text { methoxyphenyl) benzofuran, } \\
\text { 2,3'-dihydroxy-5'-methoxystilbene, } \\
\text { 2-(5'-hydroxy-3'-methoxyphenyl)-6- } \\
\text { hydroxy- 5-methoxybenzofuran, } \\
\text { 2,3'-dihydroxy-5,5'- } \\
\text { dimethoxystilbene, } \\
\text { trans-pinostilbene, }\end{array}$ & NCI-H187 & Cytotoxic effect (5.10-168.02 $\mu \mathrm{M})$ & [190] \\
\hline $\begin{array}{l}\text { Dendrobium findlayanum } \\
\text { Par. \& Rchb.f }\end{array}$ & Stems & $\begin{array}{c}\text { (R)-3, } \alpha \text {-dihydroxy- } 4,4^{\prime}, 5 \\
\text {-trimethoxybibenzyl., 3, 4-dihydroxy- } \\
3^{\prime}, 4^{\prime}, 5 \text {-Trimethoxybibenzyl, } 3^{\prime}, 4- \\
\text { dihydroxy-3, 5-dimethoxy bibenzyl, } 4 \\
4^{\prime} \text { - dihydroxy-3, 3', 5-trimethoxy } \\
\text { bibenzyl, 3, 3'-dihydroxy-5-methoxy } \\
\text { bibenzyl, 3, 3'- dihydroxy- } \\
4,5^{\prime} \text {-dimethoxy bibenzyl, } 4 \\
4^{\prime} \text {-dihydroxy-3, 5-dimethoxy bibenzyl }\end{array}$ & A172, SHSY5Y, and Hela & Cytotoxic effect $(1.65-50 \mu \mathrm{M})$ & [191] \\
\hline Dendrobium falconeri Hook. f. & Aerial parts & Dendrofalconerol A & $\mathrm{H} 460$ & Cytotoxic effect $(0.5-5 \mu \mathrm{M})$ & [192] \\
\hline Dendrobium nobile Lindl. & Stems & $\begin{array}{c}\text { dendronbibisline } \mathrm{D} \text {, dendronbibisline } \\
\mathrm{C} \text {, dendronbibisline } \mathrm{B}, \\
\text { dendronbibisline } \mathrm{A}\end{array}$ & HepG2 & $\begin{array}{c}\text { Cytotoxic effect }(1.25,4.81 \\
11.99,19.47 \mu \mathrm{M})\end{array}$ & [193] \\
\hline $\begin{array}{l}\text { Eulophia macrobulbon } \\
\text { (C.S.P.Parish \& Rchb.f.) } \\
\text { Hook.f. }\end{array}$ & Roots & $\begin{array}{c}\text { 4-methoxy-9,10-dihydro-2,7- } \\
\text { phenanthrenediol, 4-methoxy-2,7- } \\
\text { phenanthrenediol, } \\
\text { 1,5-dimethoxy-2,7-phenanthrenediol, } \\
\text { 1,5,7-trimethoxy-2,6- } \\
\text { phenanthrenediol, } \\
\text { 1-(4-hydroxybenzyl)- } \\
\text { 4,8-dimethoxy-2,7-phenanthrenediol }\end{array}$ & HeLa, CaCo-2 and MCF-7 & Cytotoxic effect $(17-100 \mu \mathrm{g} / \mathrm{mL})$ & [194] \\
\hline
\end{tabular}


Table 2. Cont.

\begin{tabular}{|c|c|c|c|c|c|}
\hline Name of Species & Part of the Plant & Active Compounds/Isolated & Cancer Cell Line & $\begin{array}{c}\text { Cytotoxic Effect/ } \\
\text { Mechanism of Action }\end{array}$ & Ref \\
\hline $\begin{array}{l}\text { Cremastra appendiculata } \\
\text { (D. Don) Makino }\end{array}$ & Tubers & Cremaphenanthrene L (1)-P & $\begin{array}{l}\text { HCT-116, Hela, MCF-7 and } \\
\text { MDA-MB-231 }\end{array}$ & $\begin{array}{l}\text { Cytotoxic effect }((1) \\
15.84-68.81 \mu \mathrm{M})\end{array}$ & [195] \\
\hline Dendrobium nobile Lindl. & Stems & $\begin{array}{c}\text { decumbic acid A, decumbic acid B, (-)- } \\
\text { decumbic acid, (-)- and (+)-dendrolactone, } \\
\text { 4-(3-hydroxyphenyl)-2- butanone, 3-hydroxy- } \\
\text { 1(3-methoxy-4-hydroxyphenyl)-propan1-one, } \\
\text { 3', } 4^{\prime}, 5^{\prime} \text {,-trimethoxycinnamyl acetate }\end{array}$ & HeLa, MCF-7 and A549 & $\begin{array}{c}\text { Cytotoxic effect } \\
\text { (from } 15.3 \text { to } 30.0 \mu \mathrm{M} \text { ) }\end{array}$ & [196] \\
\hline $\begin{array}{l}\text { Dendrobium findlayanum } \\
\text { Par. et Rchb. f }\end{array}$ & Stems & dendrobine-type alkaloids & $\begin{array}{l}\text { HL60, SMMC-7721, A-549 } \\
\text { and MCF-7 }\end{array}$ & Cytotoxic effect ( above $40 \mu \mathrm{M}$ ) & [197] \\
\hline $\begin{array}{l}\text { Arundina graminifolia } \\
\text { (D.Don) Hochr. }\end{array}$ & Aerial parts & $\begin{array}{c}\text { s 7-hydroxy-2,9-dimethoxy-1,4- } \\
\text { phenanthrenequinone named arundiquinone, } \\
\text { 5,7-dimethoxy-9,10-dihydrophenanthrene-1,2- } \\
\text { diol, rac-syringaresinol, } \\
\text { ephemeranthoquinone, coelonin }\end{array}$ & PC12 & Cytotoxic effect (about $50 \mu \mathrm{M}$ ) & [198] \\
\hline $\begin{array}{l}\text { Liparis nervosa } \\
\text { (Thunb. ex A. Murray) Lindl. }\end{array}$ & Whole plant & nervosine VII, nervosine VIII and nervosine IX & A549, MCF-7and H460 & Cytotoxic effect ( >100 mmol/L) & [199] \\
\hline Pholidota chinensis Lind. & Whole plant & polysaccharide & Caco-2 & Cytotoxic effect $(69.54 \mu \mathrm{g} / \mathrm{mL})$ & [200] \\
\hline Dendrobium plicatile Lindl. & Aerial parts & $\begin{array}{c}\text { 2-chloro-3, } \\
\text { 4'-dihydroxy-3',5-dimethoxybibenzyl, } \\
\text { 3-methylgiganto (1), } \\
\text { 3'-hydroxy-3,4,4',5-tetramethoxybibenzyl, } \\
\text { batatasinIII, moscatilin, erianthridin, coelonin, } \\
\text { 2,5-dihydroxy-4-methoxy-9,10- } \\
\text { dihydrophenanthrene, lusianthridin, } \\
\text { 1,4,7-trihydroxy-2-methoxy9,10- } \\
\text { dihydrophenanthrene, emphernathol A, } \\
\text { 3,7-dihydroxy-2,4-dimethoxy-9,10- } \\
\text { dihydrophenanthrene and calanhydroquinone } \\
\text { C three known phenanthrene, } \\
\text { 3,7-dihydroxy-2,4-dimethoxy-phenanthrene, } \\
\text { nudol and denthyrsinin }\end{array}$ & MDA-MB231, HepG2 and A549 & $\begin{array}{c}\text { Cytotoxic effect ((1) 3.41, 3.02, } \\
2.80 \mu \mathrm{M})\end{array}$ & [201] \\
\hline
\end{tabular}


Table 2. Cont.

\begin{tabular}{|c|c|c|c|c|c|}
\hline Name of Species & Part of the Plant & Active Compounds/Isolated & Cancer Cell Line & $\begin{array}{c}\text { Cytotoxic Effect/ } \\
\text { Mechanism of Action }\end{array}$ & Ref \\
\hline Cymbidium faberi Rolfe & Roots & $\begin{array}{c}\text { Coelonin, Shancidin, } \\
\text { 1-(4-hydroxybenzyl)-5,7-dimethoxy- } \\
\text { phenanthrene-2,6- diol, 5,7- } \\
\text { dimethoxyphenanthrene-2,6-diol }\end{array}$ & SMMC-7721, A549 and MGC80-3 & $\begin{array}{c}\text { Cytotoxic effect (Shancidin 12.57, } \\
18.21,11.60 \mu \mathrm{M})\end{array}$ & {$[165]$} \\
\hline $\begin{array}{l}\text { Dendrobium wardianum } \\
\text { Warner }\end{array}$ & Stems & $\begin{array}{c}\text { dendrocandin } \mathrm{V} \text {, phenanthrenes (denbinobin, } \\
\text { 9,10-dihydro-denbinobin, mostatin, } \\
\text { loddigesiinols A }\end{array}$ & $\begin{array}{l}\text { HL-60, A-549, SMMC-7721, } \\
\text { MCF-7, and SW-480 }\end{array}$ & Cytotoxic effect $(2.33-38.48 \mu \mathrm{M})$ & [202] \\
\hline $\begin{array}{l}\text { Dendrobium officinale } \\
\text { Kimura et. Migo }\end{array}$ & Stems & Fraction polysaccharides & MDA-MB-231, A549 and HepG2 & Cytotoxic effect $(0.25-3 \mathrm{mg} / \mathrm{mL})$ & [203] \\
\hline $\begin{array}{l}\text { Dendrobium officinale } \\
\text { Kimura et. Migo }\end{array}$ & Leaves & polysaccharides & U2OS and Saos-2 & $\begin{array}{c}\text { Cytotoxic effect (ranged } 12.5,25, \\
50,100, \text { and } 200 \mu \mathrm{g} / \mathrm{mL} \text { ) } \\
\text { induced cell apoptosis mediated } \\
\text { by the mitochondrial pathway by } \\
\text { up-regulating P53, Bax, and Bak } \\
\text { expression; down-regulating } \\
\text { Bcl-2 and Mcl-1 expression; and } \\
\text { increasing Cleaved } \\
\text { caspase9/Caspase9, Cleaved } \\
\text { caspase3/Caspase3, and Cleaved } \\
\text { PARP/PARP ratio }\end{array}$ & [204] \\
\hline Dendrobium offcinale Lindl. & Stems & Polysaccharide fraction & HepG2 & $\begin{array}{l}\text { Cytotoxic effect }(400 \mu \mathrm{g} / \mathrm{mL}) \\
\text { Fraction decreased the expression } \\
\text { level of Bcl-2 and increased that } \\
\text { of Bax in HepG2 cells }\end{array}$ & [205] \\
\hline $\begin{array}{l}\text { Dendrobium venustum } \\
\text { Teijsm. \& Binn. }\end{array}$ & Whole plant & Phoyunnanin E & H460, H292, and A549 & $\begin{array}{l}\text { Compound inhibit the motility of } \\
\text { lung cancer cells via the } \\
\text { suppression of EMT and } \\
\text { metastasis-related integrins }\end{array}$ & [206] \\
\hline Dendrobium offcinale Lindl. & Stems & $\begin{array}{c}\text { dendrocandin } \mathrm{P} 1 \text {, dendrocandin } \mathrm{P} 2, \\
\text { ephemeranthol A, orchinol, } \\
\text { 2, 4, 7-trihydroxy-9, 10-dihydrophenanthrene, } \\
\text { confusarin, gigantol and tristin }\end{array}$ & HL-60 and THP-1 & $\begin{array}{c}\text { Strongest cytotoxic effect } \\
\text { (orchinol values of } 11.96 \text { and } \\
8.92 \mu \mathrm{M} \text { ) }\end{array}$ & [207] \\
\hline
\end{tabular}


Table 2. Cont.

\begin{tabular}{|c|c|c|c|c|c|}
\hline Name of Species & Part of the Plant & Active Compounds/Isolated & Cancer Cell Line & $\begin{array}{c}\text { Cytotoxic Effect/ } \\
\text { Mechanism of Action }\end{array}$ & Ref \\
\hline $\begin{array}{l}\text { Nervilia concolor } \\
\text { (Blume) Schltr. }\end{array}$ & Whole plant & 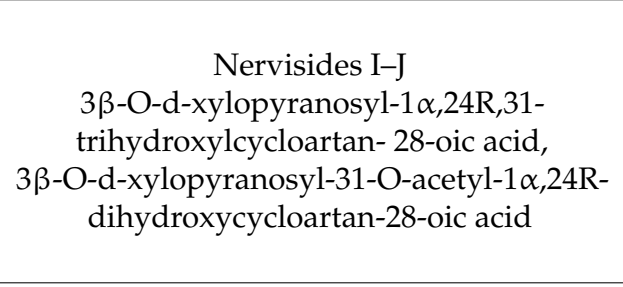 & K562 and MCF-7 & $\begin{array}{c}\text { Cytotoxic effect (Both } \\
\text { compounds } 1 \text { and } 2 \text { exerted } \\
\text { moderate activity against these } \\
\text { two cancer cell lines, with } \\
\text { respective values of } 20.5 \text { and } 20.6 \\
\mu \mathrm{g} / \mathrm{mL} \text { for } 1 \text { and } 40.1 \text { and } \\
90.5 \mu \mathrm{g} / \mathrm{mL} \text { for } 2\end{array}$ & [208] \\
\hline $\begin{array}{l}\text { Dendrobium aurantiacum var. } \\
\text { denneanum (Kerr) Z.H. Tsi }\end{array}$ & - & Moscatilin & $\begin{array}{l}\text { MG-63, A549, SK-N-SH, HCT116, } \\
\text { HeLa, HepG2, Panc-1 and BxPc-3 }\end{array}$ & $\begin{array}{l}\text { Cytotoxic effect }(25 \mu \mathrm{M} \text {, the } \\
\text { strongest effect for } \\
\text { pancreatic cells) } \\
\text { Compound induced apoptosis of } \\
\text { pancreatic cancer cells via } \\
\text { reactive oxygen species and the } \\
\text { JNK/SAPK pathway }\end{array}$ & [209] \\
\hline $\begin{array}{l}\text { Goodyera schlechtendaliana } \\
\text { Reichb.f. }\end{array}$ & Whole grass & Goodyschle A & SGC-7901 and HepG2 & $\begin{array}{l}\text { Cytotoxic effect (74.9 and } \\
89.80 \mu \mathrm{M}, \text { respectively) }\end{array}$ & [210] \\
\hline Dendrobium nobile Lindl. & Stems & nobilin $\mathrm{E}$, dendrocandin $\mathrm{V}$ & $\begin{array}{c}\text { SGC-7901, K562, A549, BEL-7402, } \\
\text { and Hela }\end{array}$ & $\begin{array}{c}\text { Cytotoxic effect (Nobilin E values } \\
\text { of } 17.30,10.39,29.03,20.13 \text {, and } \\
22.19 \mu \mathrm{M} \text {, respectively) and } \\
\text { cytotoxic effects against K562 } \\
\text { with } 28.23 \mu \mathrm{M} \text { for } \\
\text { dendrocandin V }\end{array}$ & [212] \\
\hline $\begin{array}{l}\text { Dendrobium infundibulum } \\
\text { (Lindl.) Kuntze }\end{array}$ & Whole plant & Ephemeranthol A & NCI-H460 & Cytotoxic effect $(100 \mu \mathrm{M})$ & [169] \\
\hline Cattleya tigrina A. Rich. & Whole plant & $\begin{array}{l}\text { triterpene 24-methylenecycloartanol, } \\
\text { gigantol, phocantone }\end{array}$ & HeLa & $\begin{array}{c}\text { Cytotoxic effect } \\
(86.43-90.67 \mu \mathrm{g} / \mathrm{mL})\end{array}$ & [166] \\
\hline
\end{tabular}


Table 2. Cont.

\begin{tabular}{|c|c|c|c|c|c|}
\hline Name of Species & Part of the Plant & Active Compounds/Isolated & Cancer Cell Line & $\begin{array}{c}\text { Cytotoxic Effect/ } \\
\text { Mechanism of Action }\end{array}$ & Ref \\
\hline Dendrobium draconis Rchb.f. & Stems & Batatasin III & NCI-H460 & $\begin{array}{c}\text { Cytotoxic effect }(25-100 \mu \mathrm{M}) \text { after } \\
48 \mathrm{~h} \text {. Inhibition of cell } \\
\text { proliferation }(25-100 \mu \mathrm{M}), \\
\text { migration and invasion by } \\
\text { suppressing EMT and } \\
\text { FAK/AKT/CDC42 pathway. }\end{array}$ & [108] \\
\hline Dendrobium draconis Rchb.f. & Stems & Gigantol & NCI-H460 & $\begin{array}{l}\text { Cytotoxic effect }(50 \mu \mathrm{M}) \text {. } \\
\text { Reduction of } \\
\text { anchorage-independent growth } \\
\text { and in the survival of the cancer } \\
\text { cells. Reduction in the ability of } \\
\text { the cancer cells to form tumor } \\
\text { spheroids, a critical hallmark of } \\
\text { CSCs. Reduction of lung CSCs } \\
\text { markers, including CD133 and } \\
\text { ALDH1A1. Decrease stemness in } \\
\text { the cancer cells by suppressing } \\
\text { the activation of protein kinase B } \\
\text { (Akt) signal which decreased the } \\
\text { cellular levels of pluripotency } \\
\text { and self-renewal factors Oct4 } \\
\text { and Nanog. }\end{array}$ & [211] \\
\hline Dendrobium draconis Rchb. f. & Stems & Gigantol & NCI-H460 & $\begin{array}{l}\text { Cytotoxic effect }(50 \mu \mathrm{M}) \text {. } \\
\text { Attenuation of the EMT process } \\
\text { in lung cancer cells. The } \\
\text { reduction of AKT activity. } \\
\text { Decreased transcription and the } \\
\text { stability of Slug. Reduction of } \\
\beta \text {-catenin activity and Slug } \\
\text { transcription. Enhancing GSK-3 } \beta \\
\text { ubiquitination of Slug, resulting } \\
\text { in decreased Slug levels and } \\
\text { thereby suppressing the } \\
\text { EMT process }\end{array}$ & [213] \\
\hline
\end{tabular}


Table 2. Cont.

\begin{tabular}{|c|c|c|c|c|c|}
\hline Name of Species & Part of the Plant & Active Compounds/Isolated & Cancer Cell Line & $\begin{array}{c}\text { Cytotoxic Effect/ } \\
\text { Mechanism of Action }\end{array}$ & Ref \\
\hline $\begin{array}{l}\text { Dendrobium venustum } \\
\text { Teijsm. \& Binn. }\end{array}$ & Whole plant & Phoyunnanin E & NCI-H460 & $\begin{array}{l}\text { Cytotoxic effect }(25.7 \mu \mathrm{M}) \text {. } \\
\text { Induction of apoptosis indicated } \\
\text { by condensed and fragmented } \\
\text { nuclei with the activation of } \\
\text { caspase-3 and - } 9 \text { and poly } \\
\text { (ADP-ribose) polymerase } \\
\text { cleavage. Phoyunnanin E } \\
\text { mediated apoptosis via a p53- } \\
\text { dependent pathway by } \\
\text { increasing the accumulation of } \\
\text { cellular p53 protein. Depletion of } \\
\text { antiapoptotic proteins including } \\
\text { MCL1 and Bcl2, upregulation of } \\
\text { Bax protein. Reduction in the } \\
\text { survival of cells. }\end{array}$ & [134] \\
\hline Dendrobium densiflorum Lindl. & Whole plant & Cypripedin & NCI-H460 & $\begin{array}{l}\text { The induction of apoptosis at a } \\
\text { concentration of }>50 \mu \mathrm{M} \text { with the } \\
\text { appearance of morphological } \\
\text { changes, including DNA } \\
\text { condensation and chromatin } \\
\text { fragmentation. Activation of } \\
\text { caspase- } 3 \text { and downregulation of } \\
\text { the Bcl- } 2 \text { and Bcl-xL. }\end{array}$ & [167] \\
\hline
\end{tabular}


Table 2. Cont.

\begin{tabular}{|c|c|c|c|c|c|}
\hline Name of Species & Part of the Plant & Active Compounds/Isolated & Cancer Cell Line & $\begin{array}{c}\text { Cytotoxic Effect/ } \\
\text { Mechanism of Action }\end{array}$ & Ref \\
\hline $\begin{array}{c}\text { Dendrobium } \\
\text { infundibulum Lindl. }\end{array}$ & Whole plant & Ephemeranthol A & NCI-H460 & $\begin{array}{l}\text { Cytotoxic effect }(>50 \mu \mathrm{M}) \text {. } \\
\text { Concentration-dependent cell } \\
\text { apoptosis. At non-toxic } \\
\text { concentrations inhibition of } \\
\text { anchorage-independent growth } \\
\text { of the cancer cells, as indicated } \\
\text { by the decreased colony size and } \\
\text { number. Ephemeranthol A also } \\
\text { had an inhibitory effect on } \\
\text { migration. We further found that } \\
\text { ephemeranthol A exerts its } \\
\text { antimetastatic effects via } \\
\text { inhibition of EMT, as indicated } \\
\text { by the marked decrease in } \\
\text { N-cadherin, vimentin, and Slug. } \\
\text { Furthermore, this compound } \\
\text { suppressed the activation of focal } \\
\text { adhesion kinase (FAK) and } \\
\text { protein kinase B (Akt) proteins, } \\
\text { which are key regulators of cell } \\
\text { migration. As for the anticancer } \\
\text { activity, ephemeranthol A } \\
\text { induced apoptosis by decreasing } \\
\text { Bcl-2 followed by the activation } \\
\text { of caspase } 3 \text { and caspase } 9 .\end{array}$ & [169] \\
\hline $\begin{array}{l}\text { Dendrobium officinale } \\
\text { Kimura et. Migo }\end{array}$ & Leaves & ViceninII & A549 and H1299 & $\begin{array}{c}\text { Cytotoxic effect effect }(>10 \mu \mathrm{M}) \text {. } \\
\text { ViceninII targets } \\
\text { the TGF-_/Smad } \\
\text { and PI3K/Akt/mTOR pathways } \\
\text { and inhibit TGF-1-induced EMT } \\
\text { phenotypes in lung } \\
\text { adenocarcinoma A549 and } \\
\text { H1299 cells. }\end{array}$ & [177] \\
\hline
\end{tabular}


Table 2. Cont.

\begin{tabular}{|c|c|c|c|c|c|}
\hline Name of Species & Part of the Plant & Active Compounds/Isolated & Cancer Cell Line & $\begin{array}{c}\text { Cytotoxic Effect/ } \\
\text { Mechanism of Action }\end{array}$ & Ref \\
\hline $\begin{array}{l}\text { Liparis nervosa } \\
\text { (Thunb.) Lindl. }\end{array}$ & Whole plant & Nervosine VII & HCT116 & $\begin{array}{l}\text { Cytotoxic effect ( } 11.27 \text { to } 33.8 \\
\left.\mu \text { mol. } \mathrm{L}^{-1}\right) \text {. Apoptosis associated } \\
\text { with the activation of an intrinsic } \\
\text { pathway by caspase- } 9,-3 \text { and }-7 . \\
\text { Autophagy- increase of LC3-II } \\
\text { and beclin } 1 \text { proteins, and the } \\
\text { decrease of p62 protein. } \\
\text { Induction autophagy and } \\
\text { apoptosis activated by MAPKs } \\
\text { signalling pathway including } \\
\text { JNK, ERK1/2 and p38, } \\
\text { suppressing the p53 } \\
\text { signalling pathway. }\end{array}$ & [184] \\
\hline $\begin{array}{l}\text { Dendrobium venustum } \\
\text { Teijsm. \& Binn. }\end{array}$ & Whole plant & phoyunnanin $\mathrm{E}$ & H460, H292 and A549 & $\begin{array}{l}\text { Cytotoxic effect (50 to } 100 \mu \mathrm{M}) \\
\text { Inhibition of the motility of lung } \\
\text { cancer cells via the suppression } \\
\text { of EMT and } \\
\text { metastasis-related integrins. }\end{array}$ & [206] \\
\hline
\end{tabular}




\section{In Vivo Studies of Extracts and Pure Compounds from the Orchidaceae Family}

In vivo studies are the next important step after in vitro and involve testing compounds and assessing the safety of their efficacy on living organisms such as animals, plants or whole cells. The Orchidaceae family is a valuable source of secondary metabolites (selected presented on Figure 2), and despite the limited number of studies meeting our criteria, this is also applicable to the in vivo studies which are presented below.<smiles>COc1ccc(CCc2cc(OC)c(O)c3c2C(Cc2ccc(OC)cc2)c2cc(O)c(O)c(OC)c2O3)cc1</smiles>

Dendrofalconerol A

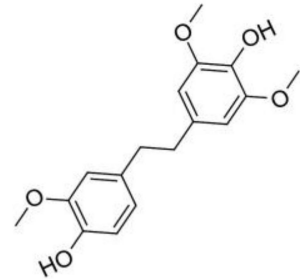

Moscatilin

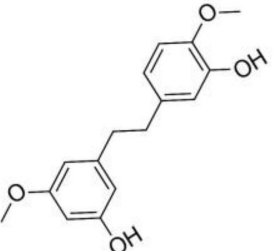

Gigantol

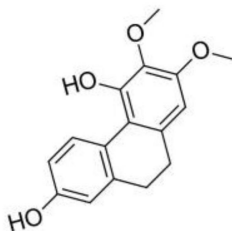

Ephemeranthol A

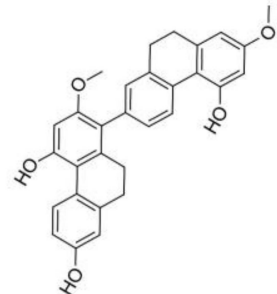

Phoyunnanin E

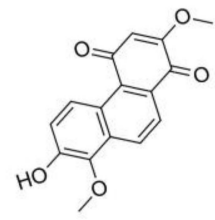

Cypripedin

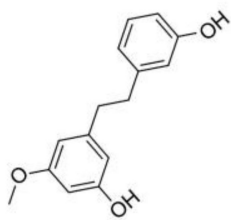

Batatasin III

Figure 2. Selected compounds from the Orchidaceae family with anticancer activity.

In the in vivo studies, Su et al. [214] evaluated the antitumour effects of moscatilin, a natural compound isolated from the orchid Dendrobium moscatum in the mouse xenograft model. MDA-MB-231 cells were axillary injected into nude mice to establish the mouse model of breast cancer. These data suggested that moscatilin suppresses breast cancer growth and progression in vivo, and therefore can be used as a potential therapeutic agent for the treatment of breast cancer [214]. Sun et al. investigated the possibility of erianin (a natural compound derived from Dendrobium candidum), as a potential therapy in colorectal cancer (CRC). The authors tested the function of erianin on tumor growth in a mouse model by injection of SW480 cells into NOD/SCID mice. These data indicated that erianin inhibited tumor growth via $\beta$-catenin in vivo [215]. On the other hand, Zhang et al. investigated the inhibitory effect of Dendrobium officinale polysaccharide (DOPA) on human gastric cancer cell SGC-7901 xenografts in nude mice, where the nude mice with SGC-7901 xenografts were randomly divided into model, 5-fluorouracil (5-Fu), low-dose DOPA, middle-dose DOPA, and high-dose DOPA group. DOPA inhibited the growth of SGC-7901 cell xenografts in nude mice. The authors suspect that the mechanism may be related to its increase of serum TNF- $\alpha$ and IL-2 levels, up-regulation of Bax protein expression, and down-regulation of Bcl-2 protein expression [216]. Zhao et al. tested Dendrobium officinale extracts ( 4.8 and $2.4 \mathrm{~g} / \mathrm{kg}$ ) which were administered orally to rats from the gastric carcinogenesis model. Compared to the cancer model group, the high-dose of Dendrobium officinale extracts significantly inhibited the rate of carcinogenesis. Further analysis showed that Dendrobium officinale extracts regulated DNA damage, oxidative stress, and carcinogenesis-related cytokines, and induced cell apoptosis to prevent gastric cancer [217]. Song et al. noted that dendrobine (an alkaloid isolated from Dendrobium nobile) enhanced the chemotoxicity of cisplatin against A549 xenograft tumor female BALB/c mice. Treatment with dendrobine or cisplatin resulted in an obvious reduction of tumour size, 
whereas combination treatment dramatically decreased the tumor size. Additionally, the authors showed that dendrobine chemo-sensitized A549 cells to cisplatin induced apoptosis through the JNK/p38 pathway in vivo [176]. In turn, Fang et al. investigated if polysaccharides isolated from Rhizoma pleionis (PRP) suppress $\mathrm{H} 22$ tumor growth in vivo in a model of malignant ascites in BALB/c mice. H22 cells were transplanted into the left abdominal cavity of mice, and then animals were treated either with PRP in saline at various doses (75, 150 , and $300 \mathrm{mg} / \mathrm{kg}$ ) or with cyclophosphamide (CTX) $(20 \mathrm{mg} / \mathrm{kg})$ or cyclophosphamide (CTX) $(20 \mathrm{mg} / \mathrm{kg})$. The authors revealed that on the tenth day after tumor cell inoculation, the mouse abdominal perimeter and weight in the PRP treatment group were significantly smaller than those in the control group. Collectively, these results demonstrated that PRP has significant antitumour properties in the H22 tumor model [218]. Other studies in xenograft analysis showed that chrysotoxene (phenanthrene derivative that was first isolated from Dendrobium chrysotoxum $)(20 \mathrm{mg} / \mathrm{kg})$ indicated that it significantly $(p<0.01)$ the inhibited growth of HepG2 cell-induced tumors by regulating the aforementioned apoptotic proteins (Smac, Cytochrome c, Survivin, Bcl-2, Bax, Apaf-1, c-caspase-9, and c-caspase-3), compared with the control group. Finally, the authors suggested that chrysotoxene may be a potential candidate drug for treating patients with hepatoblastoma [219]. Biswas et al. showed that Bulbophyllum sterile petroleum ether fraction ameliorates tumour progression in Ehrlich ascites carcinoma model in vivo. The authors revealed that the petroleum fraction of bulbs (PFB) and petroleum fraction of roots (PFR) at the dose of $200 \mathrm{mg} / \mathrm{kg}$ reduced the body weight compared to control. Cisplatin, which served as control, was injected on the first day and reduced the increase in body weight as compared to control. Additionally, the results suggested that the active fractions of bulbs and roots possess anticancer activity, likely by inducing apoptosis through the phospho-p53 dependent pathway [137]. A similar antitumour effect in an in vivo model was also shown by Jia et al. These results of antitumour activity demonstrated that the tumor weight of mice in three different dosage groups was significantly lower than that of the model group $(p<0.05, p<0.01)$. Moreover, the authors exhibited that the polysaccharide from the fibrous root of Bletilla striata had a significant inhibitory effect on the tumor growth on $\mathrm{S}_{180}$ tumor bearing mice. For this reason, the authors suggest that the mechanism of antitumour might be that it could enhance the immune function by regulating the levels of TNF and IL-2 in serum [220]. Kim et al. showed that dendrobine inhibited $\gamma$-irradiation-induced migration and invasion of A549 cells by suppressing sulfatase2 (SULF2) expression, thus inhibiting IR-induced signalling. To investigate the inhibitory effects of dendrobine in vivo, a mouse model of IR-induced metastasis, by injecting BALB/c nude mice with $\gamma$-irradiated A549 cells via the tail vein, has been established. These results noted that the number of pulmonary metastatic nodules in mice significantly reduced with dendrobine treatment (2 Gy/Dendrobine, $10.87 \pm 0.71$ ), by prevention of IR-induced signalling. For this reason, the authors report that this compound may serve as a therapeutic enhancer in non-small cell lung cancer (NSCLC) patients [221].

The studies presented above confirm the enormous anticancer potential of the compounds contained in this family, which makes them potential candidates for future anticancer therapies.

\section{Conclusions}

The review of the literature revealed that orchids have not been equally well studied throughout the world. The largest number of studies refers to Asian orchids, and little is known about the chemical constituents of American and African plants, except the pantropical Vanilla.

The literature reports that both extracts and pure compounds extracted from orchids have a strong cytotoxic effect on various cancer cell lines by inducing intrinsic and extrinsic apoptotic pathways. In addition, in vivo studies have shown that pure compounds or extracts can be used as a potential therapeutic agent in anti-cancer therapies. Considering the very low percentage of orchids examined in terms of their secondary metabolites, 
further analyses are very likely to reveal the existence of numerous new substances suitable for anticancer therapy.

Author Contributions: Conceptualization, M.K., T.Ś., T.K. and P.S.; Methodology, M.K., T.Ś.; Validation, M.K., T.Ś., T.K. and P.S.; Investigation, M.K., T.Ś, T.K. and P.S.; Resources, M.K.; Data Curation, M.K.; Writing - Original Draft Preparation, M.K., T.Ś.; Writing - Review \& Editing, M.K., T.Ś., T.K. and P.S.; Visualization T.Ś., T.K. and P.S.; Supervision, T.Ś., M.K.; Funding Acquisition, M.K. All authors have read and agreed to the published version of the manuscript.

Funding: This research was funded by the Ministry of Education, Youth and Sports of CR within the CzeCOS program, grant number LM2018123.

Acknowledgments: We are grateful to Salon Orchidei (salonorchidei.pl) for providing photos of orchid plants. Anthony Dixon is thanked for providing language correction.

Conflicts of Interest: The authors declare no conflict of interest.

\section{References}

1. Ekor, M. The growing use of herbal medicines: Issues relating to adverse reactions and challenges in monitoring safety. Front. Pharmacol. 2014, 4. [CrossRef] [PubMed]

2. Cranz, H.; Anquez-Traxler, C. TradReg 2013: Regulation of herbal and traditional medicinal products-European and global strategies-International symposium. J. Ethnopharmacol. 2014, 158 Pt B, 495-497. [CrossRef]

3. Kroes, B.H. The legal framework governing the quality of (traditional) herbal medicinal products in the European Union. J. Ethnopharmacol. 2014, 158 Pt B, 449-453. [CrossRef]

4. Petrovska, B.B. Historical review of medicinal plants' usage. Pharmacogn. Rev. 2012, 6, 1-5. [CrossRef] [PubMed]

5. Barnes, J.; McLachlan, A.J.; Sherwin, C.M.; Enioutina, E.Y. Herbal medicines: Challenges in the modern world. Part 1. Australia and New Zealand. Expert Rev. Clin. Pharmacol. 2016, 9, 905-915. [CrossRef] [PubMed]

6. Salmerón-Manzano, E.; Garrido-Cardenas, J.A.; Manzano-Agugliaro, F. Worldwide Research Trends on Medicinal Plants. Int. J. Environ. Res. Public Health 2020, 17. [CrossRef] [PubMed]

7. Gentry, A. Tropical forest biodiversity and the potential for new medicinal plants. In Human Medicinal Agents from Plants; Kinghornand, A.D., Balandrin, M.F., Eds.; American Chemical Society: Washington, DC, USA, 1993; pp. 13-24.

8. Sung, H.; Ferlay, J.; Siegel, R.; Laversanne, M.; Soerjomataram, I.; Jemal, A.; Bray, F. Global cancer statistics 2020: GLOBOCAN estimates of incidence and mortality worldwide for 36 cancers in 185 countries. CA Cancer J. Clin. 2021. [CrossRef]

9. Carelle, N.; Piotto, E.; Bellanger, A.; Germanaud, J.; Thuillier, A.; Khayat, D. Changing patient perceptions of the side effects of cancer chemotherapy. Cancer 2002, 95, 155-163. [CrossRef]

10. Luqmani, Y. Mechanisms of Drug Resistance in Cancer Chemotherapy. Med. Princ. Pract. 2005, 14, 35-48. [CrossRef]

11. Wang, Z.; Li, Y.; Ahmad, A.; Azmi, A.S.; Kong, D.; Banerjee, S.; Sarkar, F.H. Targeting miRNAs involved in cancer stem cell and EMT regulation: An emerging concept in overcoming drug resistance. Drug Resist. Update 2010, 13, 109-118. [CrossRef]

12. Ren, F.; Shen, J.; Shi, H.; Hornicek, F.; Kan, Q.; Duan, Z. Novel mechanisms and approaches to overcome multidrug resistance in the treatment of ovarian cancer. Biochim. Biophys. Acta-Rev. Cancer 2016, 1866, 266-275. [CrossRef] [PubMed]

13. Yuan, R.; Hou, Y.; Sun, W.; Yu, J.; Liu, X.; Niu, Y.; Lu, J.J.; Chen, X. Natural products to prevent drug resistance in cancer chemotherapy: A review. Ann. N. Y. Acad. Sci. 2017, 1401, 19-27. [CrossRef] [PubMed]

14. Wang, P.; Yang, H.; Yang, Y.; Wang, L.; Lee, S. Overcome Cancer Cell Drug Resistance Using Natural Products. Evid Based Complement Altern. 2015, 2015. [CrossRef] [PubMed]

15. Lin, S.; Chang, C.; Hsu, C.; Tsai, M.; Cheng, H.; Leong, M.; Sung, P.; Chen, J.; Weng, C. Natural compounds as potential adjuvants to cancer therapy: Preclinical evidence. Br. J. Pharmacol. 2020, 177, 1409-1423. [CrossRef]

16. Wang, H.; Khor, T.; Shu, L.; Su, Z.; Fuentes, F.; Lee, J.; Kong, A. Plants vs. Cancer: A Review on Natural Phytochemicals in Preventing and Treating Cancers and Their Druggability. Anti-Cancer Agents Med. Chem. 2012, 12, 1281-1305. [CrossRef] [PubMed]

17. Newman, D.J.; Cragg, G.M.; Snader, K.M. Natural products as sources of new drugs over the period 1981-2002. J. Nat. Prod. 2003, 66, 1022-1037. [CrossRef]

18. Ho, J.W.; Leung, Y.K.; Chan, C.P. Herbal medicine in the treatment of cancer. Curr. Med. Chem. Anticancer Agents 2002, 2, $209-214$. [CrossRef]

19. Musthaba, M.; Baboota, S.; Athar, T.M.; Thajudeen, K.Y.; Ahmed, S.; Ali, J. Patented herbal formulations and their therapeutic applications. Recent Pat. Drug Deliv. Formul. 2010, 4, 231-244. [CrossRef]

20. Yin, S.Y.; Wei, W.C.; Jian, F.Y.; Yang, N.S. Therapeutic applications of herbal medicines for cancer patients. Evid Based Complement Altern. Med 2013, 2013, 302426. [CrossRef]

21. Govaerts, R.; Bernet, P.; Kratochvil, K.; Gerlach, G.; Carr, G.; Alrich, P.; Pridgeon, A.M.; Pfahl, J.; Campacci, M.A.; Holland Baptista, D.; et al. World Checklist of Orchidaceae. World Checklist of Selected Plant Families. Facilitated by the Royal Botanic Gardens, Kew. 2020. Available online: http:/ / wcsp.science.kew.org (accessed on 20 November 2021). 
22. Atwood, J.T. The size of the Orchidaceae and the systematic distribution of epiphytic orchids. Selbyana 1986, 9, 171-186.

23. Joppa, L.; Roberts, D.; Pimm, S. How many species of flowering plants are there? Proc. R. Soc. B-Biol. Sci. 2011, $278,554-559$. [CrossRef] [PubMed]

24. Clements, M.; Mackenzie, A.; Copson, G.; Molloy, B.; Carmichael, N.; Skotnicki, M.; Selkirk, P. Biology and molecular phylogenetics of Nematoceras sulcatum, a second endemic orchid species from subantarctic Macquarie Island. Polar Biol. 2007, 30, 859-869. [CrossRef]

25. Dressler, R. The Orchids: Natural History and Classification; Harvard University Press: Cambridge, MA, USA, 1981.

26. Pérez-Escobar, O.A.; Chomicki, G.; Condamine, F.L.; Karremans, A.P.; Bogarín, D.; Matzke, N.J.; Silvestro, D.; Antonelli, A. Recent origin and rapid speciation of Neotropical orchids in the world's richest plant biodiversity hotspot. New Phytol. 2017, 215, 891-905. [CrossRef] [PubMed]

27. Zhang, S.; Yang, Y.; Li, J.; Qin, J.; Zhang, W.; Huang, W.; Hu, H. Physiological diversity of orchids. Plant Divers 2018, 40, 196-208. [CrossRef] [PubMed]

28. Shrestha, M.; Dyer, A.; Dorin, A.; Ren, Z.; Burd, M. Rewardlessness in orchids: How frequent and how rewardless? Plant Biol. 2020, 22, 555-561. [CrossRef]

29. Jersakova, J.; Johnson, S.; Kindlmann, P. Mechanisms and evolution of deceptive pollination in orchids. Biol. Rev. 2006, 81, 219-235. [CrossRef]

30. Johnson, S. Evidence for Batesian Mimicry In A Butterfly-Pollinated Orchid. Biol. J. Linn. Soc. 1994, 53, 91-104. [CrossRef]

31. Davies, K.L.; Roberts, D.L.; Turner, M.P. Pseudopollen and food-hair diversity in Polystachya Hook. (Orchidaceae). Ann. Bot. 2002, 90, 477-484. [CrossRef]

32. Zito, P.; Rosselli, S.; Bruno, M.; Maggio, A.; Sajeva, M. Floral scent in a sexually deceptive Ophrys orchid: From headspace collections to solvent extractions. Plant Signal Behav. 2019, 14, 1552056. [CrossRef]

33. Schiestl, F.; Ayasse, M.; Paulus, H.; Lofstedt, C.; Hansson, B.; Ibarra, F.; Francke, W. Sex pheromone mimicry in the early spider orchid (Ophrys sphegodes): Patterns of hydrocarbons as the key mechanism for pollination by sexual deception. J. Comp. Physiol. A-Neuroethol. Sens. Neural Behav. Physiol. 2000, 186, 567-574. [CrossRef]

34. Ayasse, M.; Schiestl, F.; Paulus, H.; Ibarra, F.; Francke, W. Pollinator attraction in a sexually deceptive orchid by means of unconventional chemicals. Proc. R. Soc. B-Biol. Sci. 2003, 270, 517-522. [CrossRef] [PubMed]

35. Bohman, B.; Flematti, G.; Barrow, R.; Pichersky, E.; Peakall, R. Pollination by sexual deception—It takes chemistry to work. Curr. Opin. Plant Biol. 2016, 32, 37-46. [CrossRef] [PubMed]

36. Rasmussen, H. Recent developments in the study of orchid mycorrhiza. Plant Soil 2002, 244, 149-163. [CrossRef]

37. McCormick, M.; Whigham, D.; Sloan, D.; O'Malley, K.; Hodkinson, B. Orchid-fungus fidelity: A marriage meant to last? Ecology 2006, 87, 903-911. [CrossRef]

38. Gezgin, Y.; Eltem, R. Diversity of endophytic fungi from various Aegean and Mediterranean orchids (saleps). Turk. J. Bot. 2009, 33, 439-445. [CrossRef]

39. Sarsaiya, S.; Shi, J.; Chen, J. A comprehensive review on fungal endophytes and its dynamics on Orchidaceae plants: Current research, challenges, and future possibilities. Bioengineered 2019, 10, 316-334. [CrossRef]

40. Ma, X.; Kang, J.; Nontachaiyapoom, S.; Wen, T.; Hyde, K. Non-mycorrhizal endophytic fungi from orchids. Curr. Sci. 2015, 109, 72-87.

41. Ludwig-Muller, J. Plants and endophytes: Equal partners in secondary metabolite production? Biotechnol. Lett. 2015, 37, 1325-1334. [CrossRef]

42. Bayman, P.; Gonzalez, E.; Fumero, J.; Tremblay, R. Are fungi necessary? How fungicides affect growth and survival of the orchid Lepanthes rupestris in the field. J. Ecol. 2002, 90, 1002-1008. [CrossRef]

43. McCormick, M.K.; Whigham, D.F.; Canchani-Viruet, A. Mycorrhizal fungi affect orchid distribution and population dynamics New Phytol. 2018, 219, 1207-1215. [CrossRef]

44. Zhang, F.S.; Lv, Y.L.; Zhao, Y.; Guo, S.X. Promoting role of an endophyte on the growth and contents of kinsenosides and flavonoids of Anoectochilus formosanus Hayata, a rare and threatened medicinal Orchidaceae plant. J. Zhejiang Univ. Sci. B 2013, 14, 785-792. [CrossRef] [PubMed]

45. Hinsley, A.; de Boer, H.; Fay, M.; Gale, S.; Gardiner, L.; Gunasekara, R.; Kumar, P.; Masters, S.; Metusala, D.; Roberts, D.; et al A review of the trade in orchids and its implications for conservation. Bot. J. Linn. Soc. 2018, 186, 435-455. [CrossRef]

46. Lawler, L. Ethnobotany of the Orchidaceae. In Orchid Biology, Reviews \& Perspectives III; Arditti, J., Ed.; Cornell University: Ithaca, NY, USA, 1984; pp. 27-149.

47. Teoh, E.S. Medicinal Orchids of Asia; Springer: Cham, Switzerland, 2016.

48. Bulpitt, C.J. The uses and misuses of orchids in medicine. QJM 2005, 98, 625-631. [CrossRef] [PubMed]

49. Bulpitt, C.J.; Li, Y.; Bulpitt, P.F.; Wang, J. The use of orchids in Chinese medicine. J. R Soc. Med. 2007, 100, 558-563. [CrossRef] [PubMed]

50. Kobayashi, H. Orchids as Medicinal Plants. Hortscience 2020, 55, S292.

51. Malhotra, S.; Saroya, A.; Saroya, A. Ethnopharmacology of Medicinal Orchids. In Herbalism, Phytochemistry and Ethnopharmacology.; Saroya, A., Ed.; Science Publishes: Hartford, CT, USA, 2011; pp. 300-311.

52. Chinsamy, M.; Finnie, J.; Van Staden, J. The ethnobotany of South African medicinal orchids. South African J. Bot. 2011, 77, 2-9. [CrossRef] 
53. Descourvieres, P.; Dubuisson, J.; Droissart, V.; Cribb, P.; Cawoy, V.; Simo-Droissart, M.; Sonke, B.; Stevart, T. Rhipidoglossum montealenense (Orchidaceae), a new species from Equatorial Guinea and Cameroon. Plant Ecol. Evol. 2013, 146, 389-394. [CrossRef]

54. Juswara, L.; Ormerod, P. Revision of Goodyera rubicunda (Orchidaceae: Goodyerinae). Telopea 2016, 19, 113-124. [CrossRef]

55. Vermeulen, J.; de Vogel, E.; Vogel, A. Preliminary results of an orchid survey of New Britain, Papua New Guinea: Five new species of Bulbophyllum (Orchidaceae). Blumea 2010, 55, 278-284. [CrossRef]

56. Kolanowska, M.; Baranow, P.; Nowak, S.; Fuentes, A. Materials to the revision of the genus Cranichis (Orchidaceae) in Bolivia. Phytokeys 2021, 11-41. [CrossRef]

57. Kolanowska, M.; Szlachetko, D. Crocodeilanthe (Orchidaceae) in Colombia, an underestimated diversity-New species and additions to the national orchid flora. Phyton-Ann. Rei Bot. 2020, 60, 105-144. [CrossRef]

58. Kolanowska, M.; Szlachetko, D.L.; Trejo, R.M. Telipogon diabolicus (Orchidaceae, Oncidiinae), a new species from southern Colombia. PhytoKeys 2016, 113-124. [CrossRef] [PubMed]

59. Kolanowska, M.; Szlachetko, D.; Oledrzynska, N. Ecuadorella (Orchidaceae)—The underestimated diversity. Wulfenia 2018, 25, 110-116.

60. Kolanowska, M.; Nowak, S.; Szlachetko, D. A new species of Heteranthocidium (Orchidaceae) from Ecuador. Phyton-Ann. Rei Bot. 2019, 59, 43-48. [CrossRef]

61. Kolanowska, M.; Baranow, P.; Fuentes, A. A new species of Ponthieva (Cranichidinae, Orchidaceae) from Bolivia. Phytotaxa 2019, 397, 186-192. [CrossRef]

62. Kolanowska, M.; Szlachetko, D. Notes on the genus Quekettia (Orchidaceae) with descriptions of two new species from Colombia and Guyana. Nordic J. Bot. 2019, 37. [CrossRef]

63. Kolanowska, M.; Szlachetko, D. Cranichis crenatifolia (Orchidaceae), a new species from Valle de Sibundoy, Colombia. Ann. Bot. Fenn. 2020, 57, 61-65. [CrossRef]

64. Averyanov, L.; Eskov, A.; Truong, B.; Nguyen, V.; Prilepsky, N.; Nuraliev, M.; Maisak, T.; Manh, V. The genus Octarrhena (Orchidaceae, Thelasiinae) in Vietnam with description of two new species. Phytotaxa 2020, 459, 265-275. [CrossRef]

65. Averyanov, L.; Nguyen, V.; Nguyen, K.; Maisak, T.; Truong, B. New Orchids (Orchidaceae) in the Flora of Vietnam I. Epidendroideae. Taiwania 2019, 64, 176-188. [CrossRef]

66. Averyanov, L.; Truong, B.; Nguyen, V.; Nguyen, K.; Maisak, T. New Orchids (Orchidaceae) in the Flora of Vietnam II. Vandeae. Taiwania 2019, 64, 285-298. [CrossRef]

67. Koopowitz, H.; Iamwiriyakul, P.; Laohapatcharin, S. Paphiopedilum myanmaricum, a new species of slipper orchid (Cypripedioideae, Orchidaceae). Phytotaxa 2017, 324, 97-100. [CrossRef]

68. Margonska, H. Crepidium klimkoanum-A new orchid species (Orchidaceae, Malaxidinae), from Thailand. Candollea 2005, 60, 373-377.

69. Sathapattayanon, A.; Yukawa, T.; Seelanan, T. Dendrobium roseiodorum (Orchidaceae): A new species from Vietnam. Blumea 2010, 55, 75-79. [CrossRef]

70. Chen, J.; Zhang, L.C.; Xing, Y.M.; Wang, Y.Q.; Xing, X.K.; Zhang, D.W.; Liang, H.Q.; Guo, S.X. Diversity and taxonomy of endophytic xylariaceous fungi from medicinal plants of Dendrobium (Orchidaceae). PLoS ONE 2013, 8, e58268. [CrossRef] [PubMed]

71. Sut, S.; Maggi, F.; Dall'Acqua, S. Bioactive Secondary Metabolites from Orchids (Orchidaceae). Chem. Biodivers. 2017, 14. [CrossRef] [PubMed]

72. Tupac Otero, J.; Ackerman, J.D.; Bayman, P. Diversity and host specificity of endophytic Rhizoctonia-like fungi from tropical orchids. Am. J. Bot. 2002, 89, 1852-1858. [CrossRef]

73. Bailarote, B.C.; Lievens, B.; Jacquemyn, H. Does mycorrhizal specificity affect orchid decline and rarity? Am. J. Bot. 2012, 99, 1655-1665. [CrossRef]

74. Bayman, P.; Lebron, L.; Tremblay, R.; Lodge, D. Variation in endophytic fungi from roots and leaves of Lepanthes (Orchidaceae) New Phytol. 1997, 135, 143-149. [CrossRef]

75. Xing, Y.M.; Chen, J.; Cui, J.L.; Chen, X.M.; Guo, S.X. Antimicrobial activity and biodiversity of endophytic fungi in Dendrobium devonianum and Dendrobium thyrsiflorum from Vietnam. Curr. Microbiol. 2011, 62, 1218-1224. [CrossRef]

76. Zhu, B.; Wu, L.; Wan, H.; Yang, K.; Si, J.; Qin, L. Fungal elicitors stimulate biomass and active ingredients accumulation in Dendrobium catenatum plantlets. Biologia 2018, 73, 917-926. [CrossRef]

77. Yuan, Z.; Dai, C.; Chen, L. Regulation and accumulation of secondary metabolites in plant-fungus symbiotic system. Afr. J. Biotechnol. 2007, 6, 1266-1271.

78. Cevallos, S.; Herrera, P.; Sanchez-Rodriguez, A.; Declerck, S.; Suarez, J. Untangling factors that drive community composition of root associated fungal endophytes of Neotropical epiphytic orchids. Fungal Ecol. 2018, 34, 67-75. [CrossRef]

79. Li, T.; Yang, W.; Wu, S.; Selosse, M.A.; Gao, J. Progress and Prospects of Mycorrhizal Fungal Diversity in Orchids. Front. Plant Sci. 2021, 12, 646325. [CrossRef] [PubMed]

80. Lawler, L.J.; Slaytor, M. Uses of Australian orchids by Aborigines and early settlers. Med. J. Aust. 1970, 2, 1259-1261. [CrossRef] [PubMed]

81. Teoh, E.S. Orchids as Aphrodisiac, Medicine or Food; Springer: Cham, Switzerland, 2019.

82. Pant, B.; Shah, S.; Shrestha, R.; Pandey, S.; Joshi, P.R. An Overview on Orchid Endophytes. In Mycorrhiza-Nutrient Uptake, Biocontrol, Ecorestoration; Varma, A., Prasad, R., Tuteja, N., Eds.; Springer: Cham, Switzerland, 2017; pp. 503-524. 
83. Kolanowska, M.; Konowalik, K. Niche Conservatism and Future Changes in the Potential Area Coverage of Arundina graminifolia, an Invasive Orchid Species from Southeast Asia. Biotropica 2014, 46, 157-165. [CrossRef]

84. Kolanowska, M.; Szlachetko, D. Lepanthopsis undulata (Orchidaceae), a new species from Colombia. Ann. Bot. Fenn. 2020, 57, 139-142. [CrossRef]

85. Rykaczewski, M.; Driessen, W.; Kolanowskao, M. A new species of Acianthera (Pleurothallidinae, Orchidaceae) from Brazil. Phytotaxa 2018, 372, 291-295. [CrossRef]

86. Szlachetko, D.; Chiron, G.; Kolanowska, M. A new species of Gomesa (Orchidaceae-Oncidiinae) from southern Brazil. Phyton-Ann. Rei Bot. 2018, 58, 123-125.

87. Kolanowska, M. A new species of Habenaria (Orchidaceae) from Nepal. Phytotaxa 2016, 261, 92-96. [CrossRef]

88. Kolanowska, M. Psilochilus dressleri (Orchidaceae), a new species from the Darien Gap, Panama. Phytotaxa 2014, 175, 55-58. [CrossRef]

89. Bennett, B.; Balick, M. Does the name really matter? The importance of botanical nomenclature and plant taxonomy in biomedical research. J. Ethnopharmacol. 2014, 152, 387-392. [CrossRef] [PubMed]

90. Bennett, B.C.; Balick, M.J. Phytomedicine 101: Plant taxonomy for preclinical and clinical medicinal plant researchers. J. Soc. Integr. Oncol. 2008, 6, 150-157. [PubMed]

91. Bussmann, R.W. Taxonomy-An Irreplaceable Tool for Validation of Herbal Medicine. In Evidence-Based Validation of Herbal Medicine; Mukherjee, P.K., Ed.; Elsevier: Amsterdam, The Netherlands, 2015; pp. 87-118.

92. Kolanowska, M.; Szlachetko, D. Problems with generic delimitation in the Odontoglossum complex (Orchidaceae, Oncidiinae) and an attempt for a solution. Plant Syst. Evol. 2016, 302, 203-217. [CrossRef]

93. Jakubska-Busse, A.; Zolubak, E.; Gorniak, M.; Lobas, Z.; Tsiftsis, S.; Steiu, C. A Revision of the Taxonomy and Identification of Epipactis greuteri (Orchidaceae, Neottieae). Plants 2020, 9. [CrossRef] [PubMed]

94. Pace, M.; Cameron, K. The Systematics of the Spiranthes cernua Species Complex (Orchidaceae): Untangling the Gordian Knot. Syst. Bot. 2017, 42, 1-30. [CrossRef]

95. Kolanowska, M.; Nowak, S.; Szlachetko, D. Critical notes on Draconanthes aberrans (Orchidaceae, Pleurothallidinae) with description of new species. Turk. J. Bot. 2020, 44, 192-203.

96. Feng, S.G.; Lu, J.J.; Gao, L.; Liu, J.J.; Wang, H.Z. Molecular phylogeny analysis and species identification of Dendrobium (Orchidaceae) in China. Biochem. Genet. 2014, 52, 127-136. [CrossRef]

97. Naive, M.; Handoyo, F.; Ormerod, P.; Champion, J. Dendrobium niveolabium (Orchidaceae, section Grastidium), a new Dendrobiinae species from Papua, Indonesia. Phytotaxa 2021, 490, 271-277. [CrossRef]

98. Zheng, B.; Zou, L.; Wang, X.; Wang, Y. Dendrobium jinghuanum, a new orchid species from Yunnan, China: Evidence from both morphology and DNA. Phytotaxa 2020, 428, 30-42. [CrossRef]

99. Priyadarshana, T.; Atthanagoda, A.; Wijewardhane, I.; Aberathna, N.; Peabotuwage, I.; Kumar, P. Dendrobium taprobanium (Orchidaceae): A new species from Sri Lanka with taxonomic notes on some species of the genus. Phytotaxa 2020, 432, 81-94. [CrossRef]

100. Li, H.; Xiao, W.; Tong, T.; Li, Y.; Zhang, M.; Lin, X.; Zou, X.; Wu, Q.; Guo, X. The specific DNA barcodes based on chloroplast genes for species identification of Orchidaceae plants. Sci. Rep. 2021, 11, 1421. [CrossRef] [PubMed]

101. British Columbia Ministry of Forests. Techniques and Procedures for Collecting, Preserving, Processing, and Storing Botanical Specimens; Ministry of Forests Research Program: Victoria, UK, 1996.

102. Xu, S.; Li, D.; Li, J.; Xiang, X.; Jin, W.; Huang, W.; Jin, X.; Huang, L. Evaluation of the DNA barcodes in Dendrobium (Orchidaceae) from mainland Asia. PLoS ONE 2015, 10, e0115168. [CrossRef] [PubMed]

103. Feng, S.; Jiang, Y.; Wang, S.; Jiang, M.; Chen, Z.; Ying, Q.; Wang, H. Molecular Identification of Dendrobium Species (Orchidaceae) Based on the DNA Barcode ITS2 Region and Its Application for Phylogenetic Study. Int. J. Mol. Sci. 2015, 16, 21975-21988. [CrossRef] [PubMed]

104. Guo, Y.Y.; Huang, L.Q.; Liu, Z.J.; Wang, X.Q. Promise and Challenge of DNA Barcoding in Venus Slipper (Paphiopedilum). PLoS ONE 2016, 11, e0146880. [CrossRef]

105. Wu, H.Y.; Chan, K.T.; But, G.W.; Shaw, P.C. Assessing the reliability of medicinal Dendrobium sequences in GenBank for botanical species identification. Sci. Rep. 2021, 11, 3439. [CrossRef]

106. Teoh, E.S. Secondary Metabolites of Plants. Med. Orchid. Asia 2015, 59-73.

107. Woo, K.W.; Park, J.E.; Choi, S.U.; Kim, K.H.; Lee, K.R. Phytochemical Constituents of Bletilla striata and Their Cytotoxic Activity. Nat. Prod. Sci. 2014, 20, 91-94.

108. Pinkhien, T.; Petpiroon, N.; Sritularak, B.; Chanvorachote, P. Batatasin III Inhibits Migration of Human Lung Cancer Cells by Suppressing Epithelial to Mesenchymal Transition and FAK-AKT Signals. Anticancer. Res. 2017, 37, 6281-6289. [CrossRef]

109. Tóth, B.; Hohmann, J.; Vasas, A. Phenanthrenes: A Promising Group of Plant Secondary Metabolites. J. Nat. Prod. 2018, 81, 661-678. [CrossRef]

110. Sritularak, B.; Anuwat, M.; Likhitwitayawuid, K. A new phenanthrenequinone from Dendrobium draconis. J. Asian Nat. Prod. Res. 2011, 13, 251-255. [CrossRef]

111. Bhummaphan, N.; Petpiroon, N.; Prakhongcheep, O.; Sritularak, B.; Chanvorachote, P. Lusianthridin targeting of lung cancer stem cells via Src-STAT3 suppression. Phytomedicine 2019, 62, 152932. [CrossRef] [PubMed] 
112. Wu, Y.P.; Liu, W.J.; Zhong, W.J.; Chen, Y.J.; Chen, D.N.; He, F.; Jiang, L. Phenolic compounds from the stems of Flickingeria fimbriata. Nat. Prod. Res. 2017, 31, 1518-1522. [CrossRef] [PubMed]

113. Wang, C.; Han, S.W.; Cui, B.S.; Wang, X.J.; Li, S. Chemical constituents from Pleione bulbocodioides. Zhongguo Zhong Yao Za Zhi 2014, 39, 442-447. [PubMed]

114. Liu, M.F.; Ding, Y.; Zhang, D.M. Phenanthrene constituents from rhizome of Arundina graminifolia. Zhongguo Zhong Yao Za Zhi 2005, 30, 353-356. [PubMed]

115. Guan, J.; Wang, C.L.; Xiao, P.G.; Guo, S.X. Studies on chemical components and pharmacological activities of geobiontic type medicinal plants in Orchidaceae family. Zhongguo Zhong Yao Za Zhi 2005, 30, 1053-1061.

116. Lu, T.L.; Han, C.K.; Chang, Y.S.; Lu, T.J.; Huang, H.C.; Bao, B.Y.; Wu, H.Y.; Huang, C.H.; Li, C.Y.; Wu, T.S. Denbinobin, a phenanthrene from Dendrobium nobile, impairs prostate cancer migration by inhibiting Rac1 activity. Am. J. Chin. Med. 2014, 42, 1539-1554. [CrossRef]

117. Lin, T.H.; Chang, S.J.; Chen, C.C.; Wang, J.P.; Tsao, L.T. Two phenanthraquinones from Dendrobium moniliforme. J. Nat. Prod. 2001, 64, 1084-1086. [CrossRef]

118. Gutierrez, R. Orchids: A review of uses in traditional medicine, its phytochemistry and pharmacology. J. Med. Plants Res. 2010, 4, 592-638.

119. Minh, T.N.; Khang, D.T.; Tuyen, P.T.; Minh, L.T.; Anh, L.H.; Quan, N.V.; Ha, P.T.; Quan, N.T.; Toan, N.P.; Elzaawely, A.A.; et al. Phenolic Compounds and Antioxidant Activity of Phalaenopsis Orchid Hybrids. Antioxidants 2016, 5, 31. [CrossRef]

120. Mishra, A.; Saklani, S.; Salehi, B.; Parcha, V.; Sharifi-Rad, M.; Milella, L.; Iriti, M.; Sharifi-Rad, J.; Srivastava, M. Satyrium nepalense, a high altitude medicinal orchid of Indian Himalayan region: Chemical profile and biological activities of tuber extracts. Cell. Mol. Biol. 2018, 64, 35-43. [CrossRef]

121. Favre-Godal, Q.; Gourguillon, L.; Lordel-Madeleine, S.; Gindro, K.; Choisy, P. Orchids and their mycorrhizal fungi: An insufficiently explored relationship. Mycorrhiza 2020, 30, 5-22. [CrossRef] [PubMed]

122. Pujari, I.; Thomas, A.; Rai, P.S.; Satyamoorthy, K.; Babu, V.S. In vitro bioproduction and enhancement of moscatilin from a threatened tropical epiphytic orchid, Dendrobium ovatum (Willd.) Kraenzl. 3 Biotech 2021, 11, 507. [CrossRef] [PubMed]

123. Vudala, S.M.; Padial, A.A.; Ribas, L.L.F. Micropropagation of Hadrolaelia grandis through transverse and longitudinal thin cell layer culture. S. Afr. J. Bot. 2019, 121, 76-82. [CrossRef]

124. Bhattacharyya, P.; Paul, P.; Kumaria, S.; Tandon, P. Transverse thin cell layer ( $t$-TCL)-mediated improvised micropropagation protocol for endangered medicinal orchid Dendrobium aphyllum Roxb: An integrated phytomolecular approach. Acta Physiol. Plant 2018, 40, 137. [CrossRef]

125. Mahendran, G.; Narmatha, B.V.; Akkuş, Ö.; Gürel, A. Direct somatic embryogenesis of Malaxis densiflora (A. Rich.) Kuntze. J. Genet. Eng. Biotechnol. 2016, 14,77-81. [CrossRef]

126. Ekmekçigil, M.; Bayraktar, M.; Akkuş, Ö.; Gürel, A. High-frequency protocorm-like bodies and shoot regeneration through a combination of thin cell layer and RITA ${ }^{\circledR}$ teporary immersion bioreactor in Cattleya forbesii Lindl. Plant Cell Tiss. Organ. Cult. 2019, 136, 451-464. [CrossRef]

127. Zhang, B.; Song, L.; Bekele, L.D.; Shi, J.; Jia, Q.; Zhang, B.; Jin, L.; Duns, G.J.; Chen, J. Optimizing factors affecting development and propagation of Bletilla striata in a temporary immersion bioreactor system. Sci. Hortic. 2018, 232, 121-126. [CrossRef]

128. Paudel, M.R.; Chand, M.B.; Pant, B. Antioxidant and cytotoxic activities of Dendrobium moniliforme extracts and the detection of related compounds by GC-MS. BMC Complement. Altern. Med. 2018, 18, 134. [CrossRef] [PubMed]

129. Prasad, R.; Rana, N.K.; Koch, B. Dendrobium chrysanthum ethanolic extract induces apoptosis via p53 up-regulation in HeLa cells and inhibits tumor progression in mice. J. Complement. Integr. Med. 2017, 14. [CrossRef]

130. Joshi, P.R.; Paudel, M.R.; Chand, M.B.; Pradhan, S.; Pant, K.K.; Joshi, G.P.; Bohara, M.; Wagner, S.H.; Pant, B. Cytotoxic effect of selected wild orchids on two different human cancer cell lines. Heliyon 2020, 6, e03991. [CrossRef]

131. Paudel, M.R.; Chand, M.B.; Pant, B. Assessment of Antioxidant and Cytotoxic Activities of Extracts of Dendrobium crepidatum. Biomolecules 2019, 9. [CrossRef]

132. Wang, Y.H. Traditional uses, chemical constituents, pharmacological activities, and toxicological effects of Dendrobium leaves: A review. J. Ethnopharmacol. 2021, 270, 113851. [CrossRef] [PubMed]

133. Gali-Muhtasib, H.; Hmadi, R.; Kareh, M.; Tohme, R.; Darwiche, N. Cell death mechanisms of plant-derived anticancer drugs: Beyond apoptosis. Apoptosis 2015, 20, 1531-1562. [CrossRef] [PubMed]

134. Phiboonchaiyanan, P.P.; Petpiroon, N.; Sritularak, B.; Chanvorachote, P. Phoyunnanin E Induces Apoptosis of Non-small Cell Lung Cancer Cells. Anticancer Res. 2018, 38, 6281-6290. [CrossRef] [PubMed]

135. Wu, B.; He, S.; Pan, Y.J. New dihydrodibenzoxepins from Bulbophyllum kwangtungense. Planta Med. 2006, 72, 1244-1247. [CrossRef]

136. Xu, J.; Yu, H.; Qing, C.; Zhang, Y.; Liu, Y.; Chen, Y. Two new biphenanthrenes with cytotoxic activity from Bulbophyllum odoratissimum. Fitoterapia 2009, 80, 381-384. [CrossRef]

137. Biswas, S.; Pardeshi, R.; Reddy, N.D.; Shoja, M.H.; Nayak, P.G.; Setty, M.M.; Pai, K.S.R. Bulbophyllum sterile petroleum ether fraction induces apoptosis in vitro and ameliorates tumor progression in vivo. Biomed. Pharmacother. 2016, 84, 1419-1427. [CrossRef]

138. Ye, S.; Shao, Q.; Zhang, A. Anoectochilus roxburghii: A review of its phytochemistry, pharmacology, and clinical applications. J. Ethnopharmacol. 2017, 209, 184-202. [CrossRef]

139. Liu, X.Q.; Guo, Y.Q.; Gao, W.Y.; Zhang, T.J.; Yan, L.L. Two new phenanthrofurans from Pleione bulbocodioides. J. Asian Nat. Prod. Res. 2008, 10, 453-457. [CrossRef] 
140. Wang, X.J.; Cui, B.S.; Wang, C.; Li, S. Chemical constituents from Pleione yunnanensis. Zhongguo Zhong Yao Za Zhi 2014, 39, 851-856.

141. Bhatt, D.; Jethva, K.; Zaveri, M. Phytopharmacognostical study of tubers of Eulophia nuda Lindl. Int. J. Pharm. Sci. Res. 2020, 11, 3483-3488. [CrossRef]

142. Katta, J.; Rampilla, V.; Khasim, S.M. A Study on Phytochemical and Anticancer Activities of Epiphytic Orchid Aerides odorata Lour. Eur. J. Med. Plants 2019, 28, 1-21. [CrossRef]

143. Kaliappan, V.; Kumaravelu, P. Antiproliferative effects of Vanilla planifolia leaf extract against breast cancer MCF-7 cells. Int. J. Basic Clin. Pharmacol. 2018, 8, 51-55. [CrossRef]

144. Jhansi, K.; Khasim, S.M. Antimicrobial and in vitro cytotoxic studies of Acampe praemorsa and Aeridis odarata of Orchidaceae. Ann. Plant Sci. 2018, 7, 2088-2095. [CrossRef] [PubMed]

145. Bhatt, D.R.; Jethva, K.D.; Maitreyi, N.Z. Cytotoxic activity of alcoholic extract and its fractions of Eulophia nuda tubers on MCF7 cell line. J. Pharmacogn. Phytochem. 2018, 7, 704-707.

146. Katta, J.; Rampilla, V.; Mohamad, K.S. Evaluation of phytochemical and pharmacological aspects of epiphytic orchid Luisia zeylanica Lindl. Int. J. Pharm. Sci. Res. 2020, 11, 1333-1349.

147. Ramana, N.; Ratna, K.P.; Venkateswara, R.B. GC-MS profile, antibacterial, antifungal, and anticancer activity of root of Vanda tessellata an epiphytic orchid. Int. J. Adv. Res. 2020, 8, 1553-1567. [CrossRef]

148. Soumiya, G.; Williams, B.C.; Suja, R.M. In vitro anticancer activity of ethanolic leaf extract of Acampe praemorsa (Roxb.). World J. Pharm. Res. 2018, 7, 1020-1025.

149. Haizhen, B.; Shengrong, S.; Yimin, Z.; Sun, S.; Zhongwei, C. Inhibitory effect of Dendrobium officinale polysaccharide on growth of human breast cancer MCF-7 cells and the related mechanism. Food Sci. Technol. 2017, 28, 1922-1926.

150. Bhatt, D.R.; Jethva, K.D.; Zaveri, M.N. In-vitro cytotoxicity studies of the therapeutic orchid: Eulophia nuda. J. Pharmacogn. Phytochem. 2018, 7, 680-683.

151. Nugroho, L.H.; Pratiwi, R.R.; Susandarini, R.; Wardoyo, E.; Megawati, O.; Handayan, S. Isolation of Bioactive Compounds from Two Orchid Species and Preliminary Test of Their Cytotoxicity Against T47D Breast Cancer Cells. Int. J. Pharmacogn. Phytochem. Res. 2016, 8, 150-155.

152. Jao, C.W.; Hung, T.H.; Chang, C.F.; Chuang, T.H. Chemical Constituents of Phaius mishmensis. Molecules 2016, 21, 1605. [CrossRef] [PubMed]

153. Guo, Z.; Zhou, Y.; Yang, J.; Shao, X. Dendrobium candidum extract inhibits proliferation and induces apoptosis of liver cancer cells by inactivating Wnt/ $\beta$-catenin signaling pathway. Biomed. Pharmacother. 2019, 110, 371-379. [CrossRef] [PubMed]

154. Sun, J.; Guo, Y.; Fu, X.; Wang, Y.; Liu, Y.; Huo, B.; Sheng, J.; Hu, X. Dendrobium candidum inhibits MCF-7 cells proliferation by inducing cell cycle arrest at G2/M phase and regulating key biomarkers. Oncotargets Ther. 2016, 9, 21-30. [CrossRef]

155. Prasad, R.; Koch, B. In vitro Anticancer Activities of Ethanolic Extracts of Dendrobium crepidatum and Dendrobium chrysanthum against T-cell lymphoma. J. Cytol. Histol. 2016, 7, 1000432.

156. Ho, Y.; Chen, Y.; Wang, L.; Hsu, K.; Chin, Y.; Yang, Y.; Wang, S.; Chen, Y.; Shih, Y.; Liu, L.; et al. Inhibitory Effect of Anoectochilus formosanus Extract on Hyperglycemia-Related PD-L1 Expression and Cancer Proliferation. Front. Pharmacol. 2018, 9, 807. [CrossRef]

157. Haridas, R. In-vitro cytotoxicity activity of Malaxis rheedii Sw methanol extract against hela cell line and MCF-7 cell line. Asian J. Pharm. Clin. Res. 2016, 9, 244-246. [CrossRef]

158. Liu, L.; Wang, W.; Zhao, Z.; Hu, C.; Tao, L.; Zhang, X. Pholidonone, an active stilbene derivative from Pholidota cantonensis, exhibits pro-apoptotic effect via induction of endoplasmic reticulum stress in human gastric cancer. Food Nutr. Res. $2019,63$. [CrossRef]

159. Kang, Y.Y.; Tu, Y.B.; Zhu, C.; Meng, X.F.; Yan, Y.; Wu, C.H.; Li, Y.F. Two new stilbenoids from Bletilla striata. J. Asian Nat. Prod. Res. 2019, 21, 1170-1176. [CrossRef]

160. Shao, S.Y.; Wang, C.; Han, S.W.; Sun, M.H.; Li, S. Phenanthrenequinone enantiomers with cytotoxic activities from the tubers of Pleione bulbocodioides. Org. Biomol. Chem. 2019, 17, 567-572. [CrossRef]

161. Zhang, Y.; Zhang, Q.; Xin, W.; Liu, N.; Zhang, H. Nudol, a phenanthrene derivative from Dendrobium nobile, induces cell cycle arrest and apoptosis and inhibits migration in osteosarcoma cells. Drug Des. Devel. Ther. 2019, 13, 2591-2601. [CrossRef]

162. Sun, M.H.; Ma, X.J.; Shao, S.Y.; Han, S.W.; Jiang, J.W.; Zhang, J.J.; Li, S. Phenanthrene, 9,10-dihydrophenanthrene and bibenzyl enantiomers from Bletilla striata with their antineuroinflammatory and cytotoxic activities. Phytochemistry 2021, 182, 112609. [CrossRef] [PubMed]

163. Liu, L.; Li, J.; Zeng, K.W.; Jiang, Y.; Tu, P.F. Five New Biphenanthrenes from Cremastra appendiculata. Molecules 2016, $21,1089$. [CrossRef] [PubMed]

164. Li, B.; Ali, Z.; Chan, M.; Li, J.; Wang, M.; Abe, N.; Wu, C.R.; Khan, I.A.; Wang, W.; Li, S.X. Chemical constituents of Pholidota cantonensis. Phytochemistry 2017, 137, 132-138. [CrossRef]

165. Lv, S.S.; Fu, Y.; Chen, J.; Jiao, Y.; Chen, S.Q. Six phenanthrenes from the roots of Cymbidium faberi Rolfe. and their biological activities. Nat. Prod. Res. 2020, 1-12. [CrossRef] [PubMed]

166. Ferreira, N.; Lucca, D.; Diniz, B.; Negri, M.; Milaneze-Gutierre, M.; de Oliveira, S.; Pomini, A. Chemical, chemophenetic, and anticancer studies of Cattleya tigrina. Biochem. Syst. Ecol. 2021, 97. [CrossRef] 
167. Wattanathamsan, O.; Treesuwan, S.; Sritularak, B.; Pongrakhananon, V. Cypripedin, a phenanthrenequinone from Dendrobium densiflorum, sensitizes non-small cell lung cancer H460 cells to cisplatin-mediated apoptosis. J. Nat. Med. 2018, 72, 503-513. [CrossRef] [PubMed]

168. Liao, H.F.; Pan, C.H.; Chou, P.Y.; Chen, Y.F.; Wu, T.S.; Sheu, M.J.; Wu, C.H. Toxicological effects of NCKU-21, a phenanthrene derivative, on cell growth and migration of A549 and CL1-5 human lung adenocarcinoma cells. PLoS ONE 2017, 12, e0185021. [CrossRef]

169. Nonpanya, N.; Prakhongcheep, O.; Petsri, K.; Jitjaicham, C.; Tungsukruthai, S.; Sritularak, B.; Chanvorachote, P. Ephemeranthol A Suppresses Epithelial to Mesenchymal Transition and FAK-Akt Signaling in Lung Cancer Cells. Anticancer Res. 2020, 40, 4989-4999. [CrossRef]

170. Liu, L.; Yin, Q.M.; Yan, X.; Hu, C.; Wang, W.; Wang, R.K.; Luo, X.; Zhang, X.W. Bioactivity-Guided Isolation of Cytotoxic Phenanthrenes from Spiranthes sinensis. J. Agric. Food Chem. 2019, 67, 7274-7280. [CrossRef]

171. Wang, C.; Shao, S.Y.; Han, S.W.; Li, S. Atropisomeric bi(9,10-dihydro)phenanthrene and phenanthrene/bibenzyl dimers with cytotoxic activity from the pseudobulbs of Pleione bulbocodioides. Fitoterapia 2019, 138, 104313. [CrossRef]

172. Xing, S.; Yu, W.; Zhang, X.; Luo, Y.; Lei, Z.; Huang, D.; Lin, J.; Huang, Y.; Huang, S.; Nong, F.; et al. Isoviolanthin Extracted from Dendrobium officinale Reverses TGF- $\beta 1$-Mediated Epithelial-Mesenchymal Transition in Hepatocellular Carcinoma Cells via Deactivating the TGF- $\beta$ /Smad and PI3K/Akt/mTOR Signaling Pathways. Int. J. Mol. Sci. 2018, 19, 1556. [CrossRef] [PubMed]

173. Liu, X.Q.; Li, X.P.; Yuan, W.K.; Yuan, Q.Y.; Qin, B.H. Two New Phenanthrene Glucosides from Cremastra appendiculata and their Cytotoxic Activities. Nat. Prod. Commun. 2016, 11, 477-479. [CrossRef] [PubMed]

174. Yang, D.; Cheng, Z.Q.; Yang, L.; Hou, B.; Yang, J.; Li, X.N.; Zi, C.T.; Dong, F.W.; Liu, Z.H.; Zhou, J.; et al. Seco-Dendrobine-Type Alkaloids and Bioactive Phenolics from Dendrobium findlayanum. J. Nat. Prod. 2018, 81, 227-235. [CrossRef] [PubMed]

175. Zheng, S.; Hu, Y.; Zhao, R.; Zhao, T.; Li, H.; Rao, D.; Chun, Z. Quantitative assessment of secondary metabolites and cancer cell inhibiting activity by high performance liquid chromatography fingerprinting in Dendrobium nobile. J. Chromatogr. B Analyt. Technol. Biomed. Life Sci. 2020, 1140, 122017. [CrossRef]

176. Song, T.H.; Chen, X.X.; Lee, C.K.; Sze, S.C.; Feng, Y.B.; Yang, Z.J.; Chen, H.Y.; Li, S.T.; Zhang, L.Y.; Wei, G.; et al. Dendrobine targeting JNK stress signaling to sensitize chemotoxicity of cisplatin against non-small cell lung cancer cells in vitro and in vivo. Phytomedicine 2019, 53, 18-27. [CrossRef]

177. Luo, Y.; Ren, Z.; Du, B.; Xing, S.; Huang, S.; Li, Y.; Lei, Z.; Li, D.; Chen, H.; Huang, Y.; et al. Structure Identification of ViceninII Extracted from Dendrobium officinale and the Reversal of TGF-1-Induced Epithelial-Mesenchymal Transition in Lung Adenocarcinoma Cells through TGF-/Smad and PI3K/Akt/mTOR Signaling Pathways. Molecules 2019, 24, 144. [CrossRef]

178. Chen, P.; Wu, Q.; Feng, J.; Yan, L.; Sun, Y.; Liu, S.; Xiang, Y.; Zhang, M.; Pan, T.; Chen, X.; et al. Erianin, a novel dibenzyl compound in Dendrobium extract, inhibits lung cancer cell growth and migration via calcium/calmodulin-dependent ferroptosis. Signal. Transduct. Target Ther. 2020, 5, 51. [CrossRef]

179. Zhang, Y.; Zhang, Q.; Wei, F.; Liu, N. Progressive study of effects of erianin on anticancer activity. OncoTargets Ther. 2019, 12, 5457-5465. [CrossRef]

180. Mittraphab, A.; Muangnoi, C.; Likhitwitayawuid, K.; Rojsitthisak, P.; Sritularak, B. A New Bibenzyl-phenanthrene Derivative from Dendrobium signatum and its Cytotoxic Activity. Nat. Prod. Commun. 2016, 11, 657-659.

181. Lertnitikul, N.; Pattamadilok, C.; Chansriniyom, C.; Suttisri, R. A new dihydrophenanthrene from Cymbidium finlaysonianum and structure revision of cymbinodin-A. J. Asian Nat. Prod. Res. 2020, 22, 83-90. [CrossRef]

182. Zhou, X.M.; Zheng, C.J.; Wu, J.T.; Chen, G.Y.; Zhang, B.; Sun, C.G. A new phenolic glycoside from the stem of Dendrobium nobile. Nat. Prod. Res. 2017, 31, 1042-1046. [CrossRef]

183. Yang, M.; Zhang, Y.; Chen, L.; Chen, Y. A new (propylphenyl)bibenzyl derivative from Dendrobium williamsonii. Nat. Prod. Res. 2018, 32, 1699-1705. [CrossRef] [PubMed]

184. Huang, S.; Zhao, S.M.; Shan, L.H.; Zhou, X.L. Antitumour activity of nervosine VII, and the crosstalk between apoptosis and autophagy in HCT116 human colorectal cancer cells. Chin. J. Nat. Med. 2020, 18, 81-89. [CrossRef] [PubMed]

185. Ren, G.; Deng, W.; Xie, Y.; Wu, C.; Li, W.; Xiao, C.; Chen, Y. Bibenzyl Derivatives From Leaves of Dendrobium officinale. Nat. Prod. Commun. 2020, 15. [CrossRef]

186. Liu, L.; Yin, Q.M.; Zhang, X.W.; Wang, W.; Dong, X.Y.; Yan, X.; Hu, R. Bioactivity-guided isolation of biphenanthrenes from Liparis nervosa. Fitoterapia 2016, 115, 15-18. [CrossRef]

187. Nwe, S.; Tungphatthong, C.; Laorpaksa, A.; Sritularak, B.; Thanakijcharoenpath, W.; Tanasupawat, S.; Sukrong, S. BioassayGuided Isolation of Topoisomerase I Poison from Paphiopedilum callosum (Rchb.f.) Stein. Rec. Nat. Prod. 2020, $14,89-97$. [CrossRef]

188. Li, Y.; Wang, Y.; Zhang, J. Cytotoxic Phenanthrenes and Fluorenones From Dendrobium thyrsiflorum. Chem. Nat. Compd. 2016, 52, 880-882. [CrossRef]

189. Klongkumnuankarn, P.; Busaranon, K.; Chanvorachote, P.; Sritularak, B.; Jongbunprasert, V.; Likhitwitayawuid, K. Cytotoxic and Antimigratory Activities of Phenolic Compounds from Dendrobium brymerianum. Evid. Based Complement. Altern. Med. 2015, 2015, 350410. [CrossRef]

190. Lertnitikul, N.; Jittham, P.; Khankhampoch, L.; Pattamadilok, C.; Sukrong, S.; Suttisri, R. Cytotoxic stilbenes from the roots of Paphiopedilum godefroyae. J. Asian Nat. Prod. Res. 2016, 18, 1143-1150. [CrossRef] 
191. Liu, G.Y.; Tan, L.; Cheng, L.; Ding, L.S.; Zhou, Y.; Deng, Y.; He, Y.Q.; Guo, D.L.; Xiao, S.J. Dendrobine-type alkaloids and bibenzyl derivatives from Dendrobium findlayanum. Fitoterapia 2020, 142, 104497. [CrossRef]

192. Pengpaeng, P.; Sritularak, B.; Chanvorachote, P. Dendrofalconerol A suppresses migrating cancer cells via EMT and integrin proteins. Anticancer Res. 2015, 35, 201-205.

193. Cheng, L.; Guo, D.L.; Zhang, M.S.; Linghu, L.; Fu, S.B.; Deng, Y.; He, Y.Q.; Xiao, S.J. Dihydrophenanthrofurans and bisbibenzyl derivatives from the stems of Dendrobium nobile. Fitoterapia 2020, 143, 104586. [CrossRef] [PubMed]

194. Schuster, R.; Zeindl, L.; Holzer, W.; Khumpirapang, N.; Okonogi, S.; Viernstein, H.; Mueller, M. Eulophia macrobulbon-An orchid with significant anti-inflammatory and antioxidant effect and anticancerogenic potential exerted by its root extract. Phytomedicine 2017, 24, 157-165. [CrossRef] [PubMed]

195. Liu, L.; Li, J.; Zeng, K.W.; Jiang, Y.; Tu, P.F. Five new benzylphenanthrenes from Cremastra appendiculata. Fitoterapia 2015, 103, 27-32. [CrossRef] [PubMed]

196. Zhou, X.M.; Zheng, C.J.; Wu, J.T.; Chen, G.Y.; Chen, J.; Sun, C.G. Five new lactone derivatives from the stems of Dendrobium nobile. Fitoterapia 2016, 115, 96-100. [CrossRef]

197. Yang, D.; Cheng, Z.Q.; Hou, B.; Yang, L.; Zi, C.T.; Dong, F.W.; Hu, J.M.; Zhou, J. Two unusual dendrobine-type alkaloids from Dendrobium findlayanum. Fitoterapia 2020, 144, 104607. [CrossRef]

198. Auberon, F.; Olatunji, O.J.; Krisa, S.; Antheaume, C.; Herbette, G.; Bonté, F.; Mérillon, J.M.; Lobstein, A. Two New Stilbenoids from the Aerial Parts of Arundina graminifolia (Orchidaceae). Molecules 2016, 21, 1430. [CrossRef]

199. Huang, S.; Zhong, D.; Shan, L.; Zheng, Y.; Zhang, Z.; Bu, Y.; Ma, H.; Zhou, X. Three new pyrrolizidine alkaloids derivatives from Liparis nervosa. Chin. Chem. Lett. 2016, 27, 757-760. [CrossRef]

200. Luo, D.; Wang, Z.; Li, Z.; Yu, X.Q. Structure of an entangled heteropolysaccharide from Pholidota chinensis Lindl and its antioxidant and anti-cancer properties. Int. J. Biol. Macromol. 2018, 112, 921-928. [CrossRef]

201. Chen, D.N.; Wang, Y.Y.; Liu, W.J.; Chen, Y.J.; Wu, Y.P.; Wang, J.X.; He, F.; Jiang, L. Stilbenoids from aerial parts of Dendrobium plicatile. Nat. Prod. Res. 2020, 34, 323-328. [CrossRef]

202. Zhang, C.; Liu, S.J.; Yang, L.; Yuan, M.Y.; Li, J.Y.; Hou, B.; Li, H.M.; Yang, X.Z.; Ding, C.C.; Hu, J.M. Sesquiterpene amino ether and cytotoxic phenols from Dendrobium wardianum Warner. Fitoterapia 2017, 122, 76-79. [CrossRef]

203. Wang, L.; Li, C.; Hu, C.; Gong, P.; Zhao, S. Purification and Structural Characterization of Dendrobium officinale Polysaccharides and Its Activities. Chem. Biodivers. 2021, 18. [CrossRef] [PubMed]

204. Zhang, X.; Duan, S.; Tao, S.; Huang, J.; Liu, C.; Xing, S.; Ren, Z.; Lei, Z.; Li, Y.; Wei, G. Polysaccharides from Dendrobium officinale inhibit proliferation of osteosarcoma cells and enhance cisplatin-induced apoptosis. J. Funct. Foods 2020, 73, 104143. [CrossRef]

205. Xing, S.; Zhang, X.; Ke, H.; Lin, J.; Huang, Y.; Wei, G. Physicochemical properties of polysaccharides from Dendrobium officinale by fractional precipitation and their preliminary antioxidant and anti-HepG2 cells activities in vitro. Chem. Cent. J. 2018, 12, 100. [CrossRef]

206. Petpiroon, N.; Sritularak, B.; Chanvorachote, P. Phoyunnanin E inhibits migration of non-small cell lung cancer cells via suppression of epithelial-to-mesenchymal transition and integrin $\alpha \mathrm{v}$ and integrin $\beta 3$. BMC Complement. Altern. Med. 2017, 17, 553. [CrossRef] [PubMed]

207. Zhao, G.Y.; Deng, B.W.; Zhang, C.Y.; Cui, Y.D.; Bi, J.Y.; Zhang, G.G. New phenanthrene and 9, 10-dihydrophenanthrene derivatives from the stems of Dendrobium officinale with their cytotoxic activities. J. Nat. Med. 2018, 72, 246-251. [CrossRef] [PubMed]

208. Tran, T.N.; Bernadat, G.; Mai, D.T.; Nguyen, V.K.; Sichaem, J.; Nguyen, T.P.; Tran, C.L.; Do, P.V.; Tran, N.M.; Nguyen, H.H.; et al. Nervisides I-J: Unconventional Side-Chain-Bearing Cycloartane Glycosides from. Molecules 2019, 24, 2599. [CrossRef]

209. Zhang, L.; Fang, Y.; Xu, X.; Jin, D. Moscatilin induces apoptosis of pancreatic cancer cells via reactive oxygen species and the JNK/SAPK pathway. Mol. Med. Rep. 2017, 15, 1195-1203. [CrossRef]

210. Dai, L.; Yin, Q.; Qiu, J.; Zhang, Z.; Li, G.; Huang, M.; Liu, L. Goodyschle A, a new butenolide with significant BchE inhibitory activity from Goodyera schlechtendaliana. Nat. Prod. Res. 2021, 35, 4916-4921. [CrossRef]

211. Bhummaphan, N.; Chanvorachote, P. Gigantol Suppresses Cancer Stem Cell-Like Phenotypes in Lung Cancer Cells. Evid. Based Complementary Altern. Med. 2015, 2015, 836564. [CrossRef]

212. Wang, P.; Chen, X.; Wang, H.; Huang, S.; Cai, C.; Yuan, J.; Zhu, G.; Xu, X.; Mei, W.; Dai, H. Four New Picrotoxane-Type Sesquiterpenes From Dendrobium nobile Lindl. Front. Chem. 2019, 7, 812. [CrossRef]

213. Unahabhokha, T.; Chanvorachote, P.; Sritularak, B.; Kitsongsermthon, J.; Pongrakhananon, V. Gigantol Inhibits Epithelial to Mesenchymal Process in Human Lung Cancer Cells. Evid. Based Complementary Altern. Med. 2016, 2016, 1-10. [CrossRef] [PubMed]

214. Su, W.; Zeng, L.; Chen, W. Moscatilin Suppresses the Breast Cancer Both In Vitro and In Vivo by Inhibiting HDAC3. Dose Response 2021, 19, 15593258211001251. [CrossRef] [PubMed]

215. Sun, Y.; Li, G.; Zhou, Q.; Shao, D.; Lv, J.; Zhou, J. Dual Targeting of Cell Growth and Phagocytosis by Erianin for Human Colorectal Cancer. Drug Des. Devel. Ther. 2020, 14, 3301-3313. [CrossRef] [PubMed]

216. Zhang, L.; Wang, F.; Ren, X.; Xu, G.-L.; Sun, Y.-Z.; Li, F.; Liu, H.-N. Inhibitory effect of Dendrobium officinale polysaccharide on human gastric cancer cell xenografts in nude mice. Food Sci. Technol. 2017, 38, 78-83.

217. Zhao, Y.; Liu, Y.; Lan, X.-M.; Xu, G.-L.; Sun, Y.-Z.; Li, F.; Liu, H.-N. Effect of Dendrobium officinale Extraction on Gastric Carcinogenesis in Rats. Evid. Based Complementary Altern. Med. 2016, 2016, 1213090. [CrossRef] [PubMed] 
218. Fang, Y.; Ning, A.; Li, S.; Zhou, S.; Liu, L.; Joseph, T.P.; Zhong, M.; Jiao, J.; Zhang, W.; Shi, Y.; et al. Polysaccharides Extracted from Rhizoma Pleionis Have Antitumor Properties In Vitro and in an H22 Mouse Hepatoma Ascites Model In Vivo. Int. J. Mol. Sci. 2018, 19, 1386. [CrossRef] [PubMed]

219. Dou, C.; Han, M.; Zhang, B.; Sun, L.; Jin, X.; Li, T. Chrysotoxene induces apoptosis of human hepatoblastoma HepG2 cells in vitro and in vivo via activation of the mitochondria-mediated apoptotic signaling pathway. Oncol. Lett. 2018, 15, 4611-4618. [CrossRef] [PubMed]

220. Jia, F.-H.; Tu, H.-J.; Wang, J.; Tao, G.; Ji, C.-R.; Wang, C.-X.; Xiong, F.-F.; Yan, Y.-Q. Optimizationof Ultrasonic-Flash Synergistic Extraction and Antitumor Activity of Polysaccharide from Fibrous Root of Bletilla striata. Sci. Technol. Food Ind. 2019, 40, 188-195. [CrossRef]

221. Kim, Y.-R.; Han, A.-R.; Kim, J.-B.; Jung, C.-H. Dendrobine Inhibits $\gamma$-Irradiation-Induced Cancer Cell Migration, Invasion and Metastasis in Non-Small Cell Lung Cancer Cells. Biomedicines 2021, 9, 954. [CrossRef] 